Louisiana State University

LSU Digital Commons

$9-1-2014$

\title{
Variability of optical counterparts in the Chandra galactic bulge survey
}

C. T. Britt

Louisiana State University

R. I. Hynes

Louisiana State University

C. B. Johnson

Louisiana State University

A. Baldwin

Louisiana State University

P. G. Jonker

SRON Netherlands Institute for Space Research

See next page for additional authors

Follow this and additional works at: https://digitalcommons.Isu.edu/physics_astronomy_pubs

\section{Recommended Citation}

Britt, C., Hynes, R., Johnson, C., Baldwin, A., Jonker, P., Nelemans, G., Torres, M., Maccarone, T., Steeghs, D., Greiss, S., Heinke, C., Bassa, C., Collazzi, A., Villar, A., Gabb, M., \& Gossen, L. (2014). Variability of optical counterparts in the Chandra galactic bulge survey. Astrophysical Journal, Supplement Series, 214 (1) https://doi.org/10.1088/0067-0049/214/1/10 


\section{Authors}

C. T. Britt, R. I. Hynes, C. B. Johnson, A. Baldwin, P. G. Jonker, G. Nelemans, M. A.P. Torres, T. Maccarone, D. Steeghs, S. Greiss, C. Heinke, C. G. Bassa, A. Collazzi, A. Villar, M. Gabb, and L. Gossen 


\title{
VARIABILITY OF OPTICAL COUNTERPARTS IN THE CHANDRA GALACTIC BULGE SURVEY
}

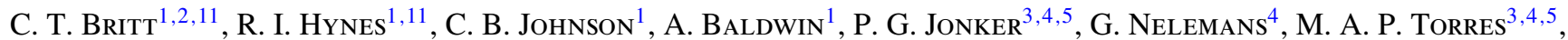

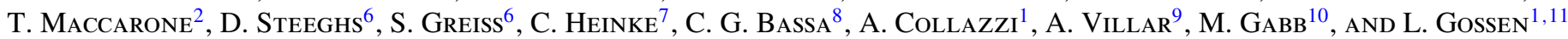 \\ ${ }^{1}$ Department of Physics and Astronomy, Louisiana State University, Baton Rouge, LA 70803-4001, USA \\ ${ }^{2}$ Department of Physics, Texas Tech University, Box 41051, Science Building, Lubbock, TX 79409-1051, USA \\ ${ }^{3}$ SRON, Netherlands Institute for Space Research, Sorbonnelaan 2, 3584 CA Utrecht, The Netherlands \\ ${ }^{4}$ Department of Astrophysics, IMAPP, Radboud University Nijmegen, Heyendaalseweg 135, 6525 AJ, Nijmegen, The Netherlands \\ ${ }^{5}$ Harvard-Smithsonian Center for Astrophysics, 60 Garden Street, Cambridge, MA 02138, USA \\ ${ }^{6}$ Astronomy and Astrophysics, Department of Physics, University of Warwick, Coventry, CV4 7AL, UK \\ ${ }^{7}$ Department of Physics, University of Alberta, CCIS 4-183, Edmonton, AB T6G 2E1, Canada \\ ${ }^{8}$ Jodrell Bank Centre for Astrophysics, School of Physics and Astronomy, University of Manchester, Manchester M13 9PL, UK \\ ${ }^{9}$ Department of Physics, Massachussettes Institute of Technology, 77 Massachusetts Avenue, Cambridge, MA 02139-4307, USA \\ ${ }^{10}$ Department of Physics, Florida Atlantic University, 777 Glades Road, Boca Raton, FL 33431-0991, USA \\ Received 2014 March 31; accepted 2014 July 3; published 2014 September 3
}

\begin{abstract}
We present optical light curves of variable stars consistent with the positions of X-ray sources identified with the Chandra X-ray Observatory for the Chandra Galactic Bulge Survey (GBS). Using data from the Mosaic-II instrument on the Blanco $4 \mathrm{~m}$ Telescope at CTIO, we gathered time-resolved photometric data on timescales from $\sim 2 \mathrm{hr}$ to 8 days over the $3 / 4$ of the X-ray survey containing sources from the initial GBS catalog. Among the light curve morphologies we identify are flickering in interacting binaries, eclipsing sources, dwarf nova outbursts, ellipsoidal variations, long period variables, spotted stars, and flare stars. Eighty-seven percent of X-ray sources have at least one potential optical counterpart. Twenty-seven percent of these candidate counterparts are detectably variable; a much greater fraction than expected for randomly selected field stars, which suggests that most of these variables are real counterparts. We discuss individual sources of interest, provide variability information on candidate counterparts, and discuss the characteristics of the variable population.
\end{abstract}

Key words: accretion, accretion disks - Galaxy: bulge - stars: black holes - stars: dwarf novae - stars: flare stars: neutron - stars: variables: general - surveys - X-rays: binaries

Online-only material: color figures, figure sets, machine-readable tables

\section{INTRODUCTION}

In the past, Galactic X-ray surveys of faint sources have focused on the Galactic center and plane, which carries the advantage of high source density (Muno et al. 2003), but also the disadvantages of high crowding and large optical extinction up to $A_{V}=30$. Together, those disadvantages make the determination of optical or infrared counterparts to X-ray sources very difficult (DeWitt et al. 2010; Mauerhan et al. 2009). Off-center multiwavelength surveys such as the ChaMPlane survey of bright X-ray sources (Grindlay et al. 2005) and the XMM-Newton Galactic Plane Survey (Hands et al. 2004) have had success in identifying significant numbers of optical/NIR counterparts in low extinction windows, typically finding large numbers of coronally active stars and active galactic nuclei (AGNs), with a few cataclysmic variables (CVs; Motch et al. 2010; Hands et al. 2004; Servillat et al. 2012; van den Berg et al. 2012). Narrow, deep surveys in the Galactic plane or bulge find even more coronal sources, as well as more CVs. (e.g., van den Berg et al. 2009). Surveys of X-ray sources in globular clusters (Heinke et al. 2003, 2005; Lu et al. 2009) avoid the problem of high extinction in the Galactic plane, but crowding is even more severe, requiring observations with Hubble Space Telescope or adaptive optics. Also, because X-ray binary formation is dominated by dynamical processes (Pooley et al. 2003; Pooley \& Hut 2006; Peacock et al. 2009), they

\footnotetext{
${ }^{11}$ Visiting astronomer, Cerro Tololo Inter-American Observatory, National Optical Astronomy Observatory, which is operated by the Association of Universities for Research in Astronomy, under contract with the National Science Foundation.
}

do not offer a probe of binary evolution in the field. Because knowledge of the counterpart is necessary for using such diagnostic tools as the ratio of X-ray to optical luminosities, ellipsoidal modulations of the companion, and optical and infrared spectroscopy, identification of counterparts is critical to the classification of the X-ray source, especially for faint X-ray sources with few detected counts. For systems accreting through Roche Lobe overflow, the masses of each component in a binary can be determined entirely by measuring the velocity amplitude of the donor star along the line of sight $K_{2}$ and the rotational broadening $v \sin i$ through spectroscopy to determine the mass ratio $q$, and the inclination angle $i$ either through the modeling of the ellipsoidal variations of the companion or the detection of eclipses.

\subsection{Galactic Bulge Survey Design, Expectations, and Goals}

The Galactic Bulge Survey (GBS; Jonker et al. 2011, 2014) is intended to avoid as much as possible the problems of crowding and extinction present in previous surveys of the Galactic center, while giving up as little as possible in the way of number of sources. The GBS makes use of both optical and X-ray imaging of two $6^{\circ} \times 1^{\circ}$ strips located 1.5 above and below the Galactic plane, cutting out the region $b<1^{\circ}$ to avoid copious amounts of dust in the Galactic plane. The GBS region and sources overlaid on the dust map from Gonzalez et al. (2012) can be seen in Figure 1. The GBS X-ray observations are short, only $2 \mathrm{ks}$, in order to keep the relative number of quiescent low mass X-ray binaries (qLMXBs) high compared to CVs and to allow a wide survey area. 


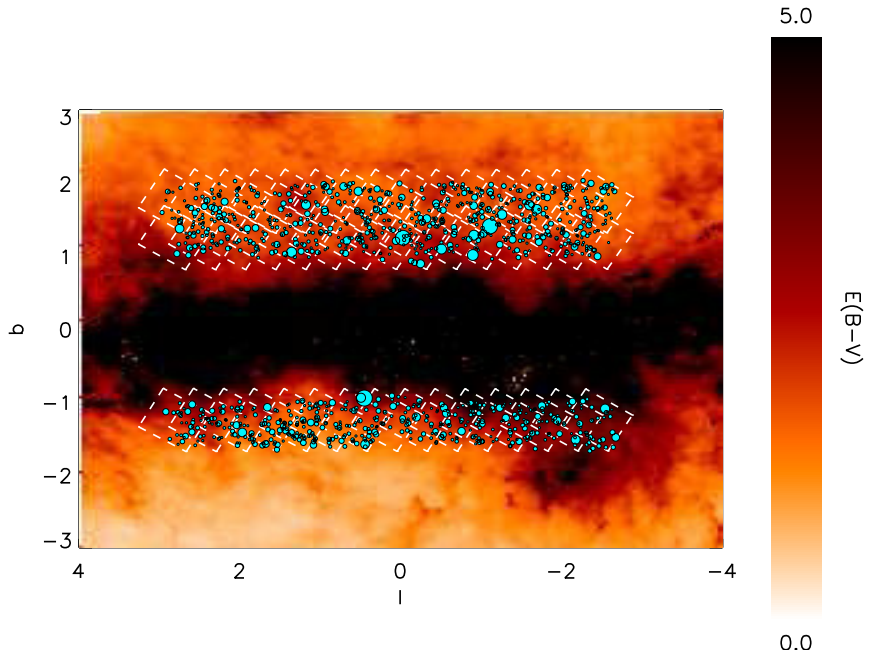

Figure 1. GBS sources overlaid on the reddening map from Gonzalez et al. (2012). The size of each point is proportional to the brightness of the X-ray source at that position. The dashed lines are the outlines of Mosaic-II fields we used for the optical side of the survey. The southern edge of the Mosaic-II region also contains some CXB sources identified in Jonker et al. (2014).

(A color version of this figure is available in the online journal.)

There are multiple science goals to be achieved in such a census of X-ray sources (Jonker et al. 2011). We aim to expand greatly the known number of Galactic X-ray binaries, including the likely discovery of the first Galactic eclipsing black hole $(\mathrm{BH})$ binary. The known population of $\mathrm{BH}$ and neutron star (NS) binaries is riddled with selection effects. For transient sources found in outburst, which includes most NS LMXBs and all dynamically confirmed BH LMXBs, the peak luminosity, outburst duration, and recurrence time depend strongly on the orbital period (Wu et al. 2010). There are no known dynamically confirmed BH LMXBs with $P_{\text {orb }}<4 \mathrm{hr}$, which is a significant paucity (Knevitt et al. 2014). There are, however, three black hole candidates (BHCs) with $P_{\text {orb }}<4 \mathrm{hr}$, identified as BHCs based on their X-ray spectra (Corral-Santana et al. 2013; Kuulkers et al. 2013; Zurita et al. 2008). Searching for LMXBs in quiescence avoids these selection effects against short period systems. By greatly increasing the number of known LMXBs, we expect to increase correspondingly the number of LMXBs for which mass determinations are possible. Mass determinations of NSs are useful in constraining the equation of state of matter at super-nuclear density in ways that cannot be done on Earth. Identification of source class types is also valuable because it allows constraints to be placed on binary evolution models by comparing observed source class numbers to the predictions of population synthesis models. Such models vary widely in their predicted number of LMXBs in the Galaxy, from $10^{3}$ to $10^{5}$ (Pfahl et al. 2003; Portegies Zwart et al. 1997; Kiel \& Hurley 2006), depending on what assumptions are made. The common envelope stage of binary evolution typically dominates the uncertainties, though other important factors include the size of supernova kicks, the initial binary fraction, the initial mass ratio distribution, and the initial orbital period distribution. Assuming a Galactic NS binary formation rate of $10^{-5} \mathrm{yr}^{-1}$ and a typical lifetime of $10^{9} \mathrm{yr}$, totalling $10^{4} \mathrm{qLMXBs}$ in the Galactic population, Jonker et al. (2011) predicted 71 qLMXBs with accessible optical counterparts $\left(r^{\prime}<23\right)$ in the GBS area, which corresponds to 53 qLMXBs in the region covered by the Mosaic-II optical observations we present here.
We expect most of these to show ellipsoidal variations in their light curves, typically of $0.1-0.2 \mathrm{mag}$.

Before any of these science goals can be achieved, it is necessary to identify properly the optical counterpart of the X-ray source. Variability is a powerful tool for ensuring proper counterpart identification. Using the Optical Gravitational Lensing Experiment (OGLE III) Catalog of Variable Stars ${ }^{12}$ (Soszyński et al. 2011a, 2011b; Szymański et al. 2011), 98\% of field stars are non-variable to $\Delta I=0.01$ in the range of $14<I<17$ photometry in the direction of the bulge, while many classes of sources that produce X-rays should also have variable optical emission. Both Udalski et al. (2012) and Hynes et al. (2012) explore the variability of possible optical counterparts to GBS sources as well, using OGLE IV and ASAS data respectively. These papers focus on somewhat brighter optical sources than are considered here. The light curve morphology also can enable determination of some system parameters, such as orbital period and inclination angle. Variability searches also can reveal the presence of high inclination systems through eclipses. Mass determinations of CVs and LMXBs are most accurate for eclipsing systems because the inclination angle is well constrained given that $\sin i \simeq 1$ as the donor eclipses either the disk or white dwarf (WD). The derived masses for eclipsers are then also relatively insensitive to inclination angle, because they depend on $\sin ^{3} i$ which is almost flat near $i=90^{\circ}$, varying by $<5 \%$ between $80^{\circ}<i<90^{\circ}$.

\subsection{Expected X-Ray Source Populations}

Jonker et al. (2011) contains a summary of the population we expect in our survey area. The total number of X-ray sources detected, 1640, very closely matches the predicted number of 1648 . We expect many different source classes which typically show variability on the timescale of hours to days. Of these, many can share light curve morphologies. For example, RS CVns and coronally active M dwarfs can show sinusoidal variations with a period of days due to star spots. These variations can be difficult to distinguish from ellipsoidal modulations when the orbital period and phasing have not been established. Although qLMXBs or CVs with main sequence (MS) donors should have periods of hours, periods greater than a day are possible for evolved donors.

The CVs in our sample are X-ray selected, and so are likely either to be magnetic systems or quiescent, as high accretion rates onto non-magnetic $\mathrm{CVs}$ create an opaque boundary layer (BL) that quenches X-ray emission, reprocessing it into UV wavelengths (Patterson \& Raymond 1985; Warner 2003). During most DN outbursts, the X-ray emission rises up until a critical value of $\dot{M}$, at which point the BL becomes opaque for the remainder of the optical outburst. There is at least one non-magnetic $\mathrm{CV}$, however, that brightens in the X-ray for the duration of the DN outbursts (i.e., U Gem, Swank et al. 1978). It is also important to note that while the X-ray emission during an outburst is typically suppressed, it does not disappear entirely. To be clear, for the purposes of this paper we consider a "quiescent" CV to be a dwarf nova in the low state, without an ionized accretion disk. New LMXBs in our survey that have optical counterparts are also likely to be in quiescence, as they are too X-ray faint to have been detected by All Sky Monitors.

Quiescent systems have a larger portion of their continuum light contributed by the donor star. In systems where the donor fills its Roche Lobe, the effective surface area of the donor

\footnotetext{
12 http://ogledb.astrouw.edu.pl/ ogle/CVS/
} 


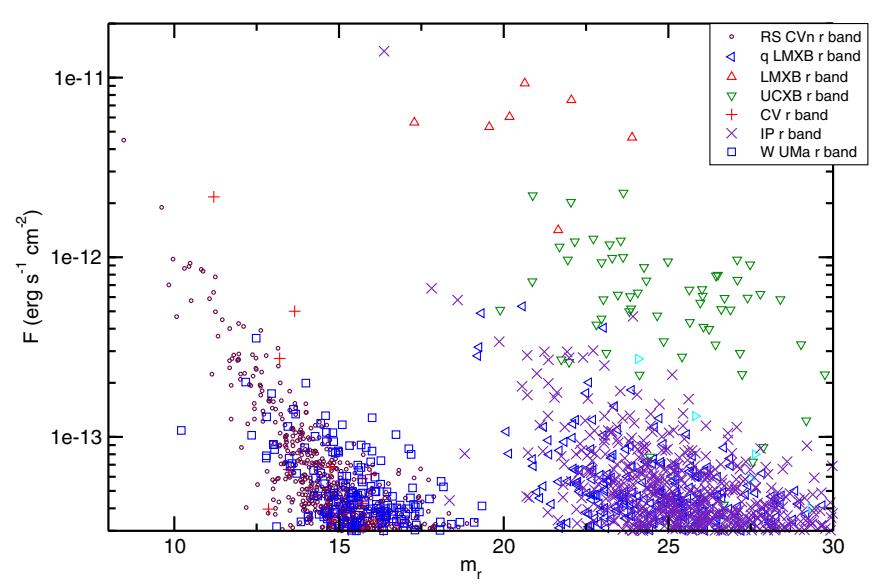

Figure 2. Estimated $r^{\prime}$ band magnitudes for the main population of GBS sources with assumptions in Jonker et al. (2011), who presented a similar figure for the Sloan $i^{\prime}$ band. Changes between the expectations in the Sloan $i^{\prime}$ band and the $r^{\prime}$ band presented here are mainly due to the difference in extinction between these two optical bands.

(A color version of this figure is available in the online journal.)

changes with phase due to tidal distortion. Because the donor contributes a large portion of the continuum light in quiescent systems, we expect to recover ellipsoidal modulations for these. Higher accretion rate systems have continuum emission dominated by the accretion disk. For systems with brighter accretion disks, we expect aperiodic flickering to dominate over any underlying periodic variations in our data set.

$\mathrm{X}$-ray detected $\mathrm{BH} \mathrm{qLMXBs}$ are likely fainter in the X-ray than NS qLMXBs at the same period because energy is carried either through the event horizon by ADAFs (Narayan et al. 1997; Garcia et al. 2001; Hameury et al. 2003; Narayan \& McClintock 2008; Rea et al. 2011) or away by jets (Fender et al. 2003). The optical light, however, emanates from the accretion disk and donor star and is comparable to NS qLMXBs at the same periods. Therefore, low ratios of X-ray to optical light somewhat favor $\mathrm{BH}$ accretors over NS accretors. It is worth noting that $L_{X} / F_{\text {opt }}$ is a rough diagnostic, representing the balance of probability rather than a hard limit between NS and $\mathrm{BH}$ qLMXBs, especially in the absence of information about the orbital period. There is therefore some overlap between NS and $\mathrm{BH}$ qLMXBs in this metric. The population of qLMXBs with $\left(L_{X} / F_{\text {opt }}\right) \approx 1$, however, should be composed primarily of NSs, and the population with $\left(L_{X} / F_{\text {opt }}\right) \approx(1 / 100)$ should host primarily BHs. We use this diagntostic to triage more intensive spectroscopic follow-up in the future.

This paper sets out to identify likely counterparts to X-ray sources in the GBS based on variability characteristics, taking into account the changing error in X-ray position with the offaxis angle of the source detection and for a different number of X-ray counts detected in the source. We identify eclipsing sources as priorities for detailed spectroscopic and X-ray follow up observations, measure the orbital periods of systems where possible, and compare the population estimates in Jonker et al. (2011), corrected for the change in filter from Sloan $i^{\prime}$ to Sloan $r^{\prime}$ and shown in Figure 2, with possible population numbers based on photometry.

\section{OBSERVATIONS AND DATA REDUCTION}

We acquired eight nights of photometry, from 2010 July 8-15, with the Blanco $4.0 \mathrm{~m}$ telescope at the Cerro Tololo InterAmerican Observatory (CTIO). Using the Mosaic-II instrument, we observed the $9 \mathrm{deg}^{2}$ area containing the X-ray sources identified by the first GBS X-ray observations (Jonker et al. 2011). At the time of the optical observations, full $X$-ray coverage of the remaining $3 \mathrm{deg}^{2}$ had not yet been approved, so we did not spend time observing the southernmost quarter of the survey area for which we had no X-ray source list. Those southern sources listed in Jonker et al. (2014) were examined with DECam in 2013 June and photometry will appear in Johnson et al. (2014). Multiple Sloan $r^{\prime}$ exposures with an integration time of $120 \mathrm{~s}$ of 45 overlapping fields were taken to cover the area. These fields are shown in Figure 1. Positions for these 45 fields were dithered between cycles to recover sources that fell on gaps between CCDs. Each field was observed 19 times over the 8 night run, but because of overlap between fields, many sources are observed at twice as many epochs, and some at half as many epochs in places with no overlap and chip gaps. The order in which the fields were cycled was randomized to minimize aliasing caused by regular sampling. Typical seeing for the run was around $1^{\prime \prime}$, though on nights 4,7 , and 8 of the observations the seeing was worse, peaking on night 8 around 2 .'5.

The data were reduced via the NOAO Mosaic Pipeline (Shaw 2009), which also added a World Coordinate System (WCS) to the images. The NOAO pipeline searches for instrumental artifacts in the image, corrects for cross talk between CCDs, applies a pupil ghost correction for light reflecting from the filter to the back surface of the corrector then back through the filter, applies bias and flat field corrections, and calibrates WCS for each image based on USNO-B1 stars in the field. Dark current calibrations are unneccesary. A detailed explanation of each procedure can be found in Chapter 2 of the NOAO Data Handbook (Shaw 2009). By comparing our fields to the UCAC3 catalog (Zacharias et al. 2009), we have found that the WCS provided by the pipeline is typically accurate to within 0.2 .

To determine the error in the X-ray position, we use the methods of Hong et al. (2005) for the 95\% confidence region. For sources at a large off-axis angle and low number of counts, this can be as large as a $10^{\prime \prime}$ radius. X-ray sources viewed close to an offset angle of zero have significantly smaller errors in the position, which can be less than $1^{\prime \prime}$ and are dominated by the boresight correction.

\subsection{Photometry}

Differential photometry was done using Alard's image subtraction routine ISIS, described in detail in Alard \& Lupton (1998) and Alard (2000). This yields changes in flux relative to the reference image. In order to convert this to magnitudes we used either aperture photometry or DAOPHOT-II (Stetson 1987) to measure the zero-point flux in the reference image. In order to save computation time, small cutouts of the full Mosaic images were taken around each object for processing. These were typically $201 \times 201$ pixels $\left(52^{\prime \prime} \times 52^{\prime \prime}\right)$ or $401 \times$ 401 pixels $\left(104^{\prime \prime} \times 104^{\prime \prime}\right)$, although in a few cases it was necessary to increase the field to $801 \times 801$ pixels if the field near the X-ray position contained many saturated stars. A subtraction was deemed to be "clean" if it resulted in a variance image free of the vast majority of field stars and free of artifacts from the wings of saturated stars near the X-ray position.

We found that different fields required somewhat different keyword values in ISIS to obtain a clean subtraction around the object of interest. For most fields, we ran ISIS with a kernel composed of 3 Gauss multiplied by polynomials of degree 6,4 , and 3 with $\sigma=1.1,3.0$, and 5.5 pixels, respectively. 
The kernel and background were also most often fit with a first-order polynomial to allow for spatial variation across the field, though this was sometimes increased to a second-order polynomial if the subtraction was not clean. Some fields contain artifacts or very bright stars in one section; for these fields we divided the image into separate parts for the image subtraction.

We adopt a definition of variability wherein an object is said to be variable either if the standard deviation is at least three times the typical relative photometric error or if there is at least one observation greater than four times its relative photometric error away from the mean. The only objects subjected to these tests are those that are at all visible in the variance image produced by ISIS and those shown in other observations to be emission line objects.

\subsection{Periodicities}

For all variable sources, periodograms were created using the Lomb-Scargle statistic (Lomb 1976; Scargle 1982) in an effort to search for periodicities. Since ellipsoidal variations have two maxima and minima in a single orbital cycle, we also folded the light curve on periods twice as long as prominent peaks on the periodogram. We also consider both aliases and harmonics, as higher harmonics can sometimes show up at a higher power than the fundamental frequency. For sources with multiple observations of an eclipse, we also searched for periods with Phase Dispersion Minimization (Stellingwerf 1978). The significance of identified periods is checked through Monte Carlo simulations in which the order of the observations is randomized. If the recovered period is due to the overfitting of Gaussian noise, then reshuffling the light curve should also provide similar fits to noise in a non-trivial number of cases. We consider periods to be significant if these searches result in less than $0.5 \%$ of simulations with a higher spectral power at any point in frequency space than the initial recovered period in the properly ordered data. Since we have 165 variable potential counterparts, we expect of the order of 1 false period detection at this threshhold.

Systems with a power density spectrum of red or pink noise can easily yield false periods that appear significant in white noise tests (Vaughan 2010), though sparse sampling means that most flickering should appear as white noise (i.e., nearby points are not correlated). It is unclear that irregular variables in our data exhibit red noise power spectra. In order to test the likelihood of spurious identification of periods in our data set, we used the existing OGLE-IV photometry for GBS sources (Udalski et al. 2012), which has a baseline $\approx 100 \times$ as long as our Mosaic-II data. Using irregular variables aligned with X-ray sources in this data set and pulling out 8 days worth of data at a time, we ran period searches and Monte Carlo simulations just as we do for the Mosaic-II data. We recover spurious periods that appear to be significant under white noise simulations approximately $6 \%$ of the time. Furthermore, of the 11 systems that both appear to be long period variables in Mosaic-II data and appear in OGLE-IV photometry, 10 are truly periodic in OGLE-IV data. We conclude that we have $\lesssim 2$ sources with spurious periods between 1 and 4 days, and that the majority of periods recovered are likely real. This assumes that the power spectra for the fainter objects is similar to that of the brighter objects.

\subsection{Magnitude Calibrations}

At present, all apparent magnitudes cited here are scaled to nearby stars in the USNO-B1 catalog (Monet et al. 2003) and are to be used with caution until secondary standards are established for all Mosaic fields. The magnitude scaling, which is a pipeline calibration product, carries an estimated uncertainty of $\pm 0.5 \mathrm{mag}$ for each source. This is adequate for estimating $\mathrm{X}$-ray to optical flux ratios, as the uncertainty is dominated by low photon counts in the X-ray, uncertainty in the X-ray spectral shape, and uncertainty in the reddening and absorption for the vast majority of sources.

\section{RESULTS}

Of the 1234 X-ray sources identified with the northern three quarters of the Chandra observations, 18 sources are likely duplicates of the same X-ray source with positions separated by $>3^{\prime \prime}$ that were not removed when the catalog was made (Hynes et al. 2012) leaving 1216 unique sources. Of these, 20 lie outside regions imaged by Mosaic-II, 255 have likely counterparts saturated in the Mosaic-II data, and a further 98 sources are too near a saturated star or a bleed trail to do photometry on. A sizable number of optically bright sources was anticipated, and many are covered by other observations (see, e.g., Hynes et al. 2012; Udalski et al. 2012), while the focus of this work was the optically faint population. This leaves 843 unique sources with useful Mosaic-II data. These comprise 111 sources with no detectable counterpart in Sloan $r^{\prime}$ inside the $95 \% \mathrm{X}$-ray confidence region, 567 that have no counterpart that shows variability over the course of our observations in the $95 \%$ confidence region, and 165 sources that have possible counterparts that show variability during our observations. The majority of variables show flickering, for which we were unable to recover a period.

\subsection{Consideration of Chance Alignments}

Inevitably, when examining 1216 unique sources, variables occasionally lie outside the $95 \%$ confidence region. In order to estimate the rate of coincidence with variable interlopers, we first estimate typical crowding in our fields by counting the number of stars in regions with a radius of $3^{\prime \prime}$ offset from a random selection of X-ray positions by $15^{\prime \prime}$. We find a mean stellar density of $0.064 \operatorname{arcsec}^{-2}$. Approximately 61 true counterparts should lie between the $95 \%$ and $3 \sigma$ confidence regions, which means we should have $\approx 9$ variable true counterparts falling outside the error region given the observed rate of variability within the $95 \%$ confidence region of GBS sources. This is less than the $\sim 40$ chance variables in the annulus between the $95 \%$ and $3 \sigma$ confidence regions that one expects by assuming that the rate of variability for interlopers down to $r^{\prime}<23$ is approximately that of the population measured by OGLE III $(I<17)$, where the probable number of variable interlopers inside a given area is a Poisson distribution. Similarly, there should be $\sim 20$ variable interlopers within $2^{\prime \prime}$ of the X-ray positions. The further from the center of the X-ray position a variable is, the more likely it is to be an interloper as the area of the sky being searched increases. We compare the likelihood of finding a true variable counterpart at a certain radius compared to the likelihood of finding a variable interloper within the same radius to assign a false alarm probability (FAP) to variables near X-ray positions, so that FAP $=\left[1-\left(2 / \sigma_{X} \sqrt{2 \pi}\right) e^{-\left(r / \sigma_{X} / 2\right)^{2} / 2}\right][1-$ $\left.e^{-\pi r^{2} \times 0.00128 \text { variables arcsec }}{ }^{-2}\right]$. For sources with more than one variable star aligned with the X-ray position, those that show light curve morphology unique to either CVs or LMXBs, such as DN outbursts, should be considered the counterpart, while variables showing more ubiquitous morphologies, such as sinusoidal or 
Table 1

The Table Below Shows the Total Breakdown of Sources in Mosaic-II Data

\begin{tabular}{lr}
\hline \hline Category & Number \\
\hline Duplicates & 18 \\
Saturated & 255 \\
Coincidentally saturated & 98 \\
Off-chip & 20 \\
Not variable & 567 \\
No counterpart $\left(r^{\prime} \lesssim 23\right)$ & 111 \\
Variable & 165 \\
\hline Types of Variability & \\
Flickering & 76 \\
Outburst/flare & 11 \\
Eclipsing & 27 \\
Ellipsoidal & 5 \\
Sinusoidal & 38 \\
Long period variables & 18 \\
\hline
\end{tabular}

Note. The largest category is non-variables, with sources either saturated or obscured by bleed trails as the second largest.

ellipsoidal modulations, should be considered to be more likely to be a chance alignment without other compelling evidence to the contrary. The number of sources we have in each category of variability is shown in more detail in Table 1.

Many sources (567) show no detectable optical variability. For some of these, the true counterpart could be below detection limits, leaving only field stars coincident with the X-ray position. There are also 111 sources with no possible counterpart in Sloan $r^{\prime}<23$. In Jonker et al. (2011), a simplistic population model predicted that the GBS would find $\sim 400$ sources in Mosaic-II fields without a counterpart in Sloan $i^{\prime}$ band, which increases in Sloan $r^{\prime}$ because of greater extinction. The predicted number is much higher than that observed, suggesting that many possible non-variable counterparts are in fact random field stars. Sources with large errors on the X-ray position admit many possibilities as counterparts, but the true counterpart may be below detection limits. There are 537 sources with an estimated error with a radius larger than $1^{\prime \prime} .9$, each of which admits probable chance alignments, assuming a Poisson distribution of stars with the measured density on the sky. Of these, 256 have at least one possible non-variable counterpart within detection limits. This suggests that hundreds of true counterparts are indeed non-variable. Some of these have been confirmed through spectroscopic studies, e.g., H $\alpha$ emitters CX561 and CX1004 (Britt et al. 2013; Torres et al. 2014). We expect to be sensitive to intrinsic ellipsoidal modulations of $0.1 \mathrm{mag}$ for more than $99 \%$ of spatially isolated objects with $r^{\prime}<19$, with sensitivity decreasing as photometric errors rise to the level of the projected variations. Because binary systems are uniformly distributed in $\cos i$ on the sky while the projected variation scales with $\sin i$, we expect to maintain sensitivity to ellipsoidal intrinsic variations of $0.1 \mathrm{mag}$ for the majority of sources until $r^{\prime} \approx 21$, when photometric errors become comparable to $1 / 3$ the rms of the ellipsoidal modulations in our line of sight.

\subsection{Sensitivities of the Survey}

The X-ray to optical flux ratio is calculated in Table 2 for sources with variable counterparts for both absorbed and unabsorbed flux at bulge distance using assumptions in Jonker et al. (2011), the extinction law $R_{V}=3.1$ found in Cardelli et al. (1989), and the relation between optical extinction and hydrogen column density $N_{\mathrm{H}}=0.58 \times 10^{22} \times E(B-V)$ from Bohlin et al. (1978). $F_{\mathrm{Opt}}=v F_{v}$ is calculated from apparent magnitudes using filter properties for Sloan $r^{\prime}$. For bulge extinction values, we use the maps from Gonzalez et al. (2012). We transform these values to the Sloan $r^{\prime}$ filter using filter properties given in Schlegel et al. (1998). Many sources are closer than the bulge distance, so the absorbed and estimated unabsorbed flux ratios represent approximate upper and lower limits, respectively, to $F_{X} / F_{\text {Opt }}$ though there is additional uncertainty in this ratio because X-ray and optical observations are not simultaneous, as well as uncertanties in both $F_{X}$ from photon noise and spectral shape and in $F_{\text {opt }}$ from the calibration of the zero point magnitude. We should not detect sources with an X-ray luminosity below $10^{32} \mathrm{erg} \mathrm{s}^{-1}$ at bulge distance, so RS CVns, W UMas, CVs, and coronally active stars should tend to be in the foreground and therefore to have a value toward the upper end of a source's range of possible flux ratios.

Table 2

Each GBS Source with a Likely Variable Optical Counterpart

\begin{tabular}{|c|c|c|c|c|c|c|c|c|c|c|c|c|c|}
\hline CX ID & R.A. & Decl. & $\begin{array}{c}r^{\prime} \\
(\mathrm{mag})\end{array}$ & $\langle$ error $\rangle$ & $\mathrm{rms}$ & $\begin{array}{c}\text { Distance } \\
(\operatorname{arcsec})\end{array}$ & $\begin{array}{c}95 \% \mathrm{CR} \\
(\operatorname{arcsec})\end{array}$ & $N$ & $\begin{array}{c}\text { Absorbed } \\
\log \frac{F_{X}}{F_{\mathrm{opt}}}\end{array}$ & $E(B-V)$ & $\log \frac{F_{X}}{F_{\mathrm{opt}}}$ & HR & FAP \\
\hline 2 & 264.36832 & -29.13384 & 18.14 & 0.004 & 0.027 & 0.192 & 0.839 & 36 & 1.9 & 1.72 & 0.3 & 0.00 & $2.12 \mathrm{E}-05$ \\
\hline 5 & 265.03806 & -28.79050 & 18.61 & 0.005 & 0.084 & 0.162 & 0.802 & 28 & 1.0 & 2.73 & -1.6 & 0.50 & $8.74 \mathrm{E}-06$ \\
\hline 11 & 265.46423 & -27.03995 & 20.70 & 0.025 & 0.164 & 1.603 & 1.605 & 33 & 1.6 & 2.10 & -0.4 & 0.64 & $9.55 \mathrm{E}-03$ \\
\hline 18 & 264.89896 & -27.49324 & 17.36 & 0.003 & 0.577 & 0.406 & 1.017 & 19 & 0.0 & 1.45 & -1.3 & -0.20 & $2.84 \mathrm{E}-04$ \\
\hline 19 & 267.47760 & -29.72652 & 19.21 & 0.007 & 0.243 & 0.763 & 1.133 & 20 & 0.8 & 1.99 & -1.0 & 0.89 & $1.67 \mathrm{E}-03$ \\
\hline 21 & 265.39072 & -28.67623 & 18.46 & 0.004 & 0.371 & 0.703 & 1.440 & 18 & 0.5 & 2.64 & -2.0 & -0.38 & $1.30 \mathrm{E}-03$ \\
\hline 23 & 265.63152 & -27.73004 & 17.96 & 0.003 & 0.025 & 0.983 & 1.115 & 36 & 0.2 & 2.06 & -1.7 & 0.39 & $3.28 \mathrm{E}-03$ \\
\hline 28 & 264.94583 & -27.30242 & 16.89 & 0.002 & 0.204 & 0.803 & 0.892 & 28 & -0.3 & 1.47 & -1.6 & 0.50 & $2.12 \mathrm{E}-03$ \\
\hline 29 & 268.42447 & -28.06488 & 16.83 & 0.001 & 0.036 & 0.520 & 0.893 & 19 & -0.3 & 2.75 & -2.9 & -0.14 & 5.92E-04 \\
\hline 37 & 264.37158 & -29.46776 & 19.33 & 0.009 & 0.187 & 0.689 & 1.424 & 33 & 0.6 & 1.89 & -1.1 & 0.90 & $1.23 \mathrm{E}-03$ \\
\hline
\end{tabular}

Notes. Only the first 10 objects are presented here. The full list can be found in the online article. The columns are as follows: (1) catalog ID, (2) Right Ascension, (3) Declination, (4) $r^{\prime}$ magnitude, (5) average relative photometric error, (6) rms variation of the light curve, (7) distance of variable star from X-ray position, (8) 95\% confidence radius of X-ray position, (9) number of observations in the light curve, (10) log of the X-ray to optical flux ratio without correcting for extinction, (11) $E(B-V)$ for the line of sight at bulge distance using the reddening maps from Gonzalez et al. (2012), (12) X-ray to optical flux ratio after correcting for reddening, assuming bulge distance, (13) X-ray hardness ratio as calculated in Jonker et al. (2011), only presented for sources with $>20$ photons, (14) false alarm probability for a variable star at the given distance from the X-ray position, as calculated in Section 3.

(This table is available in its entirety in a machine-readable form in the online journal. A portion is shown here for guidance regarding its form and content.) 

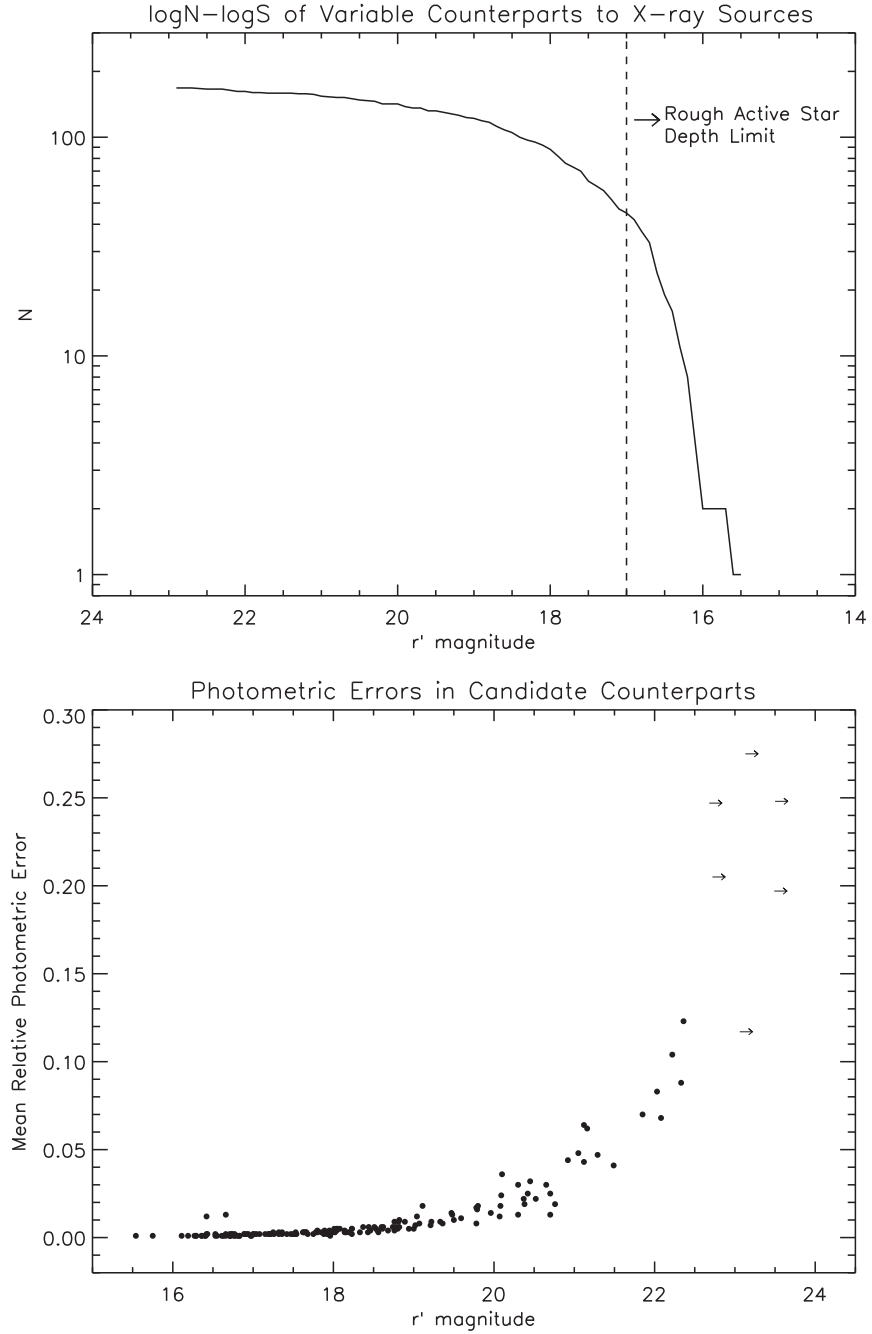

Figure 3. Top: a cumulative histogram of the mean magnitude of variable sources near X-ray positions in the GBS. The dashed line indicates the X-ray survey's detection limit of active stars assuming that they have $\left(F_{X} / F_{\text {opt }}\right)=(1 / 100)$ and $E(B-V)=0.5$. This limit is uncertain due to factors discussed in Section 3. The sudden change in slope at this point is expected because the nature of the source population should change as one goes deeper into the optical, from systems with low $F_{X} / F_{\text {opt }}$ such as RS CVns, W UMas, and other coronally active stars, to systems with higher $F_{X} / F_{\text {opt }}$ such as qLMXBs and CVs. Bottom: the mean photometric error in variable sources plotted against the mean magnitude. The relative photometric error does not become high enough to mask the expected variability from ellipsoidal modulations in qLMXBs or CVs until $r^{\prime}=21-22$, depending on the amplitude of the variations.

The sources with potential variable counterparts are listed in Table 2. Sources with rms scatter greater than three times the statistical error in the measurements are considered to be variable. Sources with at least a single data point more than $4 \sigma$ from the mean magnitude are also considered to be variable. A cumulative histogram of the mean optical magnitudes of variable counterpart candidates is shown in Figure 3. There is a pronounced knee at roughly the magnitude at which RS CVns and W UMa binaries should lie at a great enough distance to cease to be detected in the X-ray survey. The sudden change in slope in the $\log N-\log S$ distribution is attributable to the changing source population rather than a decreased discovery efficiency at that brightness. As shown in the bottom panel of Figure 3, the average relative photometric error remains low well past the magnitude at which the break occurs.

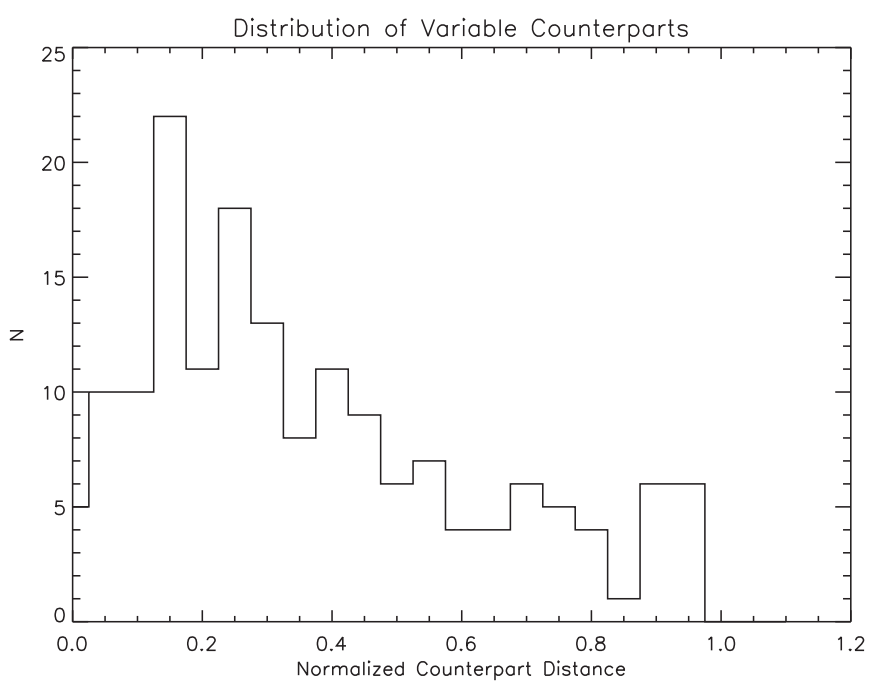

Figure 4. Histogram of the distances between variable possible counterparts relative to the $95 \%$ confidence radius of Chandra X-ray positions in Jonker et al. (2011). $72 \%$ of variable counterparts are within half the $95 \%$ confidence radius, which is a strong indicator that the vast majority of variables included here are likely true counterparts to the X-ray source. The number of interlopers should grow toward the outer edge of the error region as the area of the sky enclosed in each annulus increases.

The majority of variables identified as candidate counterparts are within $1^{\prime \prime}$ of the Chandra X-ray position. A histogram of all the offsets between the optical counterpart and the Chandra position is shown in Figure 4, normalized to the uncertainty in the $\mathrm{X}$-ray position. The fact that the variables are concentrated in the inner half of the error region suggests that the vast majority are truly associated with the X-ray source, as the effective area on the sky, and therefore the chance of variable interlopers, increases with radius. Light curve data for each variable source is available in Table 3.

\section{SELECTED SOURCES}

Finding charts and variance maps for all sources with possible counterparts can be found online, ${ }^{13}$ as can all light curves for sources with variable counterparts. Some individual sources warrant more detailed discussion. Sources with existing spectroscopy are covered in depth in Britt et al. (2013), Hynes et al. (2014), Maccarone et al. (2012), Ratti et al. (2013), Torres et al. (2014), and Wu et al. (2014). Most sources discussed here still require spectroscopy for a full classification.

\subsection{CX11-Magnetic CV or $q L M X B$}

The variability of CX11 is irregular and on the order of 0.2-0.4 mag. This object has an X-ray to optical flux ratio of 40 , uncorrected for extinction, and shows dramatic, aperiodic variations shown in Figure 5. $L_{X} \approx 10^{34}(d / 8 \mathrm{kpc})^{2} \mathrm{erg} \mathrm{s}^{-1}$ and $\left(F_{X} / F_{\text {opt }}\right)=0.4$ with bulge reddening. $F_{X} / F_{\text {opt }}$ is high enough to argue against a quiescent $\mathrm{CV}$, which are not typically luminous enough in the X-ray to be detected at this strength in the Galactic bulge and thus could not suffer the full amount of extinction predicted by the Gonzalez et al. (2012) maps, though it is consistent with a closer magnetic CV or qLMXB. In addition, CX11 is X-ray bright enough that a meaningful hardness ratio can be calculated from the 2 ks exposure 13 Available at tigers.phys.lsu.edu/gbs/vardb/ and as figure sets in the online
journal. 
Table 3

Light Curves for Each Variable Optical Counterpart to a GBS Source

\begin{tabular}{lccr}
\hline \hline CX ID & HJD & $r^{\prime}$ Mag & $r^{\prime}$ Error \\
\hline 2 & 2455386.61987 & 18.123 & 0.004 \\
2 & 2455386.63620 & 18.145 & 0.004 \\
2 & 2455386.80615 & 18.139 & 0.003 \\
2 & 2455386.84960 & 18.137 & 0.004 \\
2 & 2455387.49606 & 18.087 & 0.003 \\
2 & 2455387.61573 & 18.130 & 0.003 \\
2 & 2455387.66526 & 18.142 & 0.004 \\
2 & 2455387.72729 & 18.160 & 0.003 \\
2 & 2455387.84241 & 18.114 & 0.004 \\
2 & 2455388.52084 & 18.144 & 0.003 \\
\hline
\end{tabular}

Notes. The columns are as follows: (1) catalog ID, (2) HJD of observation, (3) $r^{\prime}$ magnitude, (4) relative photometric error.

(This table is available in its entirety in a machine-readable form in the online journal. A portion is shown here for guidance regarding its form and content.)

(as described in Jonker et al. 2011), showing that the X-ray spectrum is fairly hard with $[2.5-8]-[0.3-2.5] /[0.3-8]=$ 0.64 . This X-ray hardness further rules out a quiescent $\mathrm{CV}$ as well as arguing against thermal emission from a NS qLMXB, though some NS qLMXBs have strong power law components that would result in a hard X-ray spectrum (Wijnands et al. 2005, e.g., EXO1745-248). Using the Web-PIMMS tool, we determine that the X-ray color is too hard for quiescent CVs assuming a $10 \mathrm{keV}$ thermal Brehmsstrahlung spectral shape. As discussed in Section 1.2, BH qLMXBs tend to be fainter in X-ray than NS qLMXBs at the same period, resulting in lower values of $F_{X} / F_{\text {opt }}$. We therefore prefer a magnetic CV interpretation for CX11, though a qLMXB interpretation cannot be firmly ruled out without further data.

\section{2. $C X 19-C V$ or $q L M X B$}

CX19 also appears in OGLE-IV fields (Udalski et al. 2012), where it is observed to eclipse with a period of $P_{\text {orb }}=$ 0.3587 days. Our observations also show that CX19 eclipses, as shown in Figure 5, in addition to having a fairly high X-ray to optical flux ratio of 6 before correcting for absorption and $1 / 10$ assuming reddening for bulge distance. Either of these is consistent with a qLMXB or CV. It also shows large amplitude aperiodic variability, up to 0.4 mag, consistent with a large contribution from the accretion disk to continuum light. The eclipse is deep, almost a magnitude, implying a substantial temperature difference between bodies. The eclipse lasts no more than $3 \mathrm{hr}$, and there is only 1 observation in eclipse out of 20 . It is very likely a binary with a compact object. This object is $1^{\prime \prime} .27$ away from a star that is very red and which dominates over it in VVV data and in Two Micron Sky Survey (2MASS) data. CX19 is a candidate eclipsing qLMXB or CV. (Torres et al. 2014) classify this object spectroscopically as a high accretion rate $\mathrm{CV}$, which is consistent with our photometric classification.

$$
\text { 4.3. } C X 21-C V
$$

CX21 is strongly variable, on timescales of hours, showing a change in brightness of over 1 mag shown in Figure 5. There is no apparent periodicity to these changes. Absorbed $\left(F_{X} / F_{\text {opt }}\right)=3$ which drops to 0.01 for bulge distance reddening, a range that is consistent with CVs, qLMXBs, and AGN. $L_{X} \approx 3 \times 10^{33}(\mathrm{~d} / 8 \mathrm{kpc})^{2} \mathrm{erg} \mathrm{s}^{-1}$. The high amplitude flickering suggests a large contribution to the continuum light from the accreting material compared to the donor star, which argues weakly against a quiescent system. We can safely rule out an RS CVn, W UMa, or active star because of the strong flickering. An AGN is possible, but we consider it unlikely because of the short timescale of the variability.

CX21 is a ROSAT source (Jonker et al. 2011), which detected it in a $2418 \mathrm{~s}$ observation and saw possible evidence of variability. It is somewhat soft in the $\mathrm{X}$-ray in GBS observations with a hardness ratio of -0.38 in $[2.5-8]-[0.3-2.5] /[0.3-8.0] \mathrm{keV}$, and somewhat hard in ROSAT observations with a hardness ratio of $\mathrm{HR} 2=0.28$ in $[0.9-2.0]-[0.5-0.9] /[0.5-2.0] \mathrm{keV}$. Because these measures are of different parts of the spectrum, they do not suggest a change in the spectral shape between observations. The ROSAT observation is hard enough to argue against thermal emission from a NS qLMXB even with minimal extinction, while the hardness ratio from Chandra is too soft for a $\mathrm{BH}$ qLMXB even with maximal extinction assuming a power law spectrum with $\Gamma=2$. Both hardness ratios are consistent with thermal Brehmsstrahlung from a $\mathrm{CV}$.

\subsection{CX29-Flare Star}

CX29 shows smooth variations which both rise and fall, as shown in Figure 5. There is a crest and a trough and our data are consistent with a period of $\sim 10.2$ days, but this is highly speculative. There is a small flare, $\approx 0.1$ mag in amplitude, which lasts for several hours on the fifth night of observations. Absorbed $\left(F_{X} / F_{\text {opt }}\right)=0.5$, which is consistent with CVs, qLMXBs, and with active $\mathrm{M}$ dwarfs since most the light they emit is in the infrared so that $\left(F_{X} / F_{\text {opt }}\right) \gg\left(F_{X} / F_{\text {bol }}\right)$. $L_{X} \approx 2.4 \times 10^{33}(\mathrm{~d} / 8 \mathrm{kpc})^{2} \mathrm{erg} \mathrm{s}^{-1}$. If this were an $\mathrm{M}$ dwarf with an absolute magnitude of $M_{r^{\prime}}=15$, then the distance would be $23 \pm 7 \mathrm{pc}$ which would imply $L_{X} \approx 2 \times 10^{28} \mathrm{erg} \mathrm{s}^{-1}$ which is consistent with $M$ dwarfs. In the 2MASS survey (Skrutskie et al. 2006), this star has $J-K=1.15$ and $K=12.50$, which is consistent with a late $\mathrm{M}$ dwarf at that distance. It is possible that this is a flare star, with the complex multi-day variations arising from a combination of star spots as the star rotates with $P_{\text {spin }}>8$ days. CX29 appears in Udalski et al. (2012) with a period of 12.77 days, which is consistent with our observations. Differences among multiple star spots in phase and brightness, and even small differences in period by latitude due to differential rotation, can lead to more complex light curve morphologies such as in the case of CX29. This object is suggested to be a chromospheric active star or binary after spectroscopic observations in Torres et al. (2014) pending further analysis, which agrees with our photometric classification.

\subsection{CX67-CV or $q L M X B$}

CX67 shows variations of several hundredths of a magnitude, with a suspected period of 5.67 days. The Mosaic-II light curve is shown in Figure 5. Absorbed $\left(F_{X} / F_{\text {opt }}\right)=0.7$ which is consistent with CVs, qLMXBs, and $M$ dwarfs, but the flickering visible in the light curve argues against an $M$ dwarf interpretation. It is possible that this is a longer period binary with a compact object and a subgiant donor, such as V404 Cyg (Hynes et al. 2009). If this is the case, $F_{X} / F_{\text {opt }}$ should be lower for quiescent systems than for systems with a MS donor because the optical contribution from the larger counterpart is much higher. The amplitude of variations is quite low; if these variations are due to tidal distortion of the donor, the inclination angle of the system must be fairly low as well. 

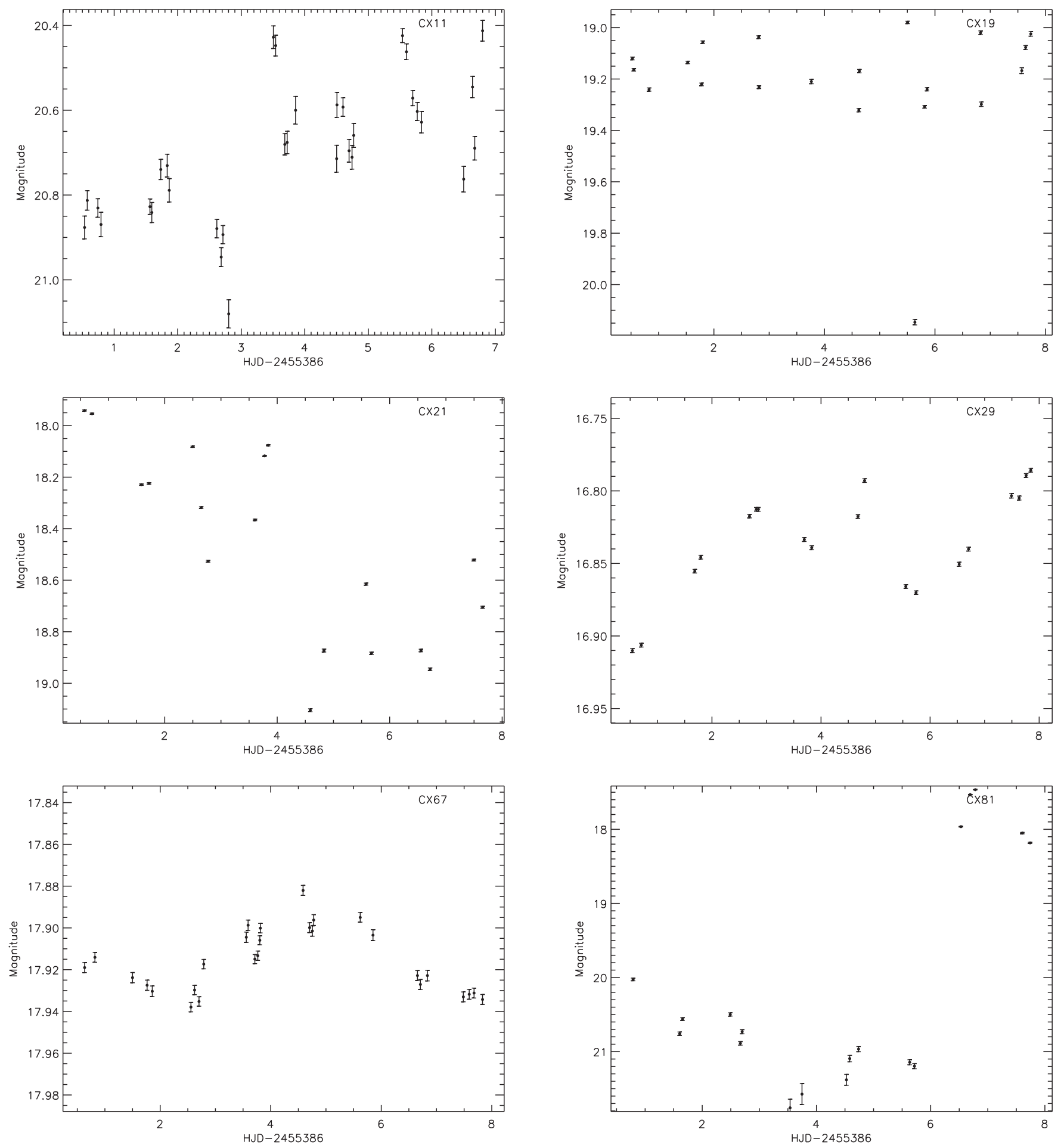

Figure 5. CX11, CX19, CX21, CX29, CX67, CX81 Mosaic-II light curves.

\section{6. $C X 81-D N$}

CX81 shows an outburst of $3.5 \mathrm{mag}$ and lasts at least a few days, as shown in Figure 5, which is typical of DNe. Absorbed $\left(F_{X} / F_{\text {opt }}\right)=5$ in quiescence, which is consistent with CVs. This source is very likely a CV undergoing DN outbursts.

\section{7. $C X 83-C V$ or $q L M X B$}

CX83 is not significantly variable except for a possible eclipse on the 5 th night of observations shown in Figure 6. This eclipse is $0.2 \mathrm{mag}$ in depth and lasts no more than $2 \mathrm{hr}$. Only one observation out of 26 points is in eclipse which lasts only $4 \% \pm 4 \%$ of the orbital phase, assuming that we evenly sample all phases. From these constraints, we place an upper limit on the orbital period of 2 days. Absorbed $\left(F_{X} / F_{\text {opt }}\right)=7$, which drops to 0.02 with reddening at the bulge distance, which is a range consistent with both CVs and qLMXBs. The X-ray spectrum is very hard, with a hardness ratio of 0.86 , which is consistent with up-scattering from a disk corona at high inclination and not with the thermal emission expected for a quiescent $\mathrm{CV}$, so a qLMXB interpretation is favored. 

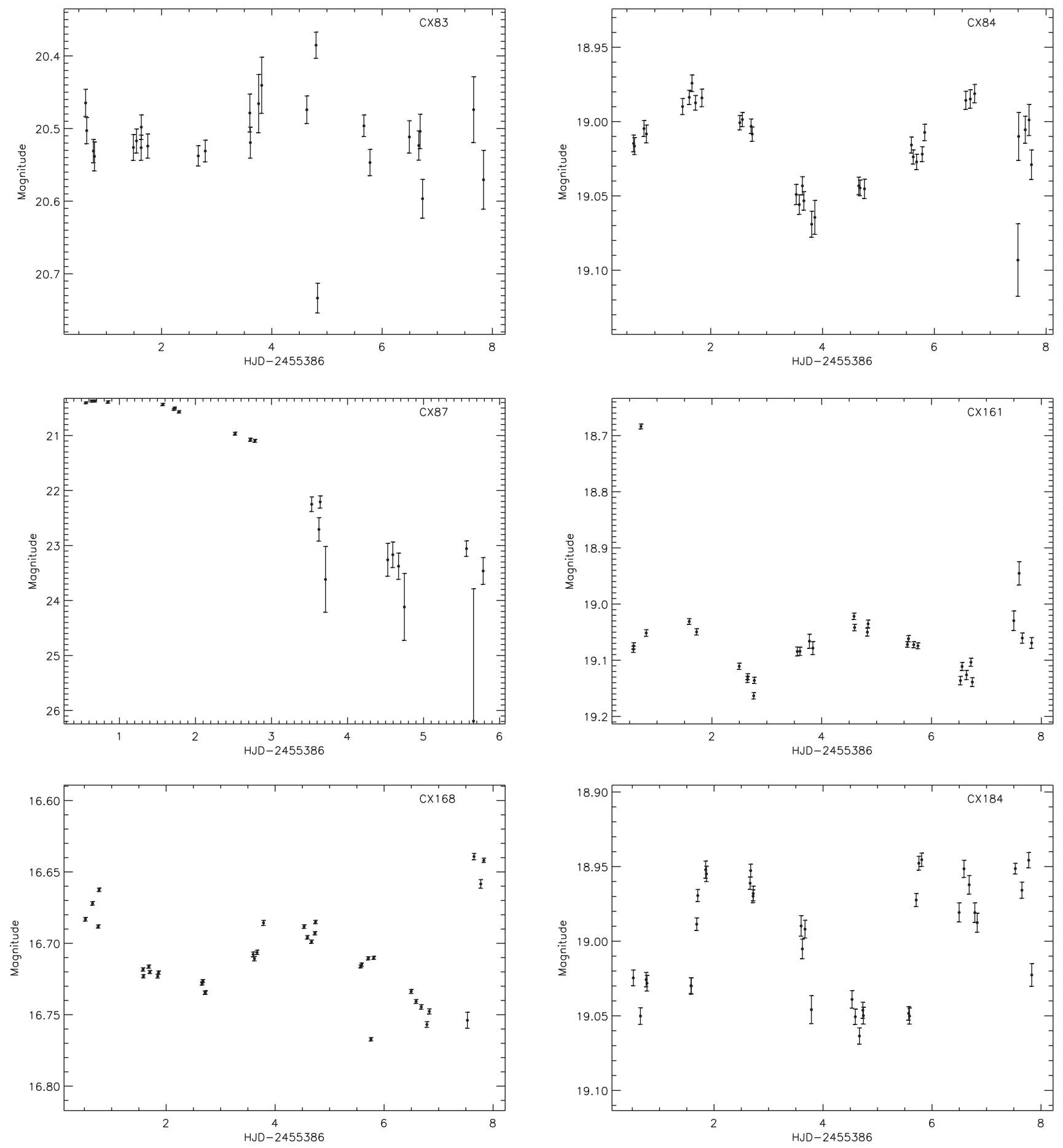

Figure 6. CX83, CX84, CX87, CX161, CX168, CX184 Mosaic-II light curves.

\subsection{CX84-CV or $q L M X B$}

CX84 has a suspected period of 4.67 days shown in Figure 6, and an absorbed $\left(F_{X} / F_{\text {opt }}\right)=2$. The amplitude of the variations is $0.1 \mathrm{mag}$ which is consistent with both ellipsoidal variations from accreting binaries. Similarly to CX67, this object is unlikely to be an RS CVn based on the high X-ray to optical flux ratio, but it could be a qLMXB or CV with an evolved donor because of the multi-day orbital period. In the direction of the bulge, $E(B-V)=1.98$, which implies $M_{r^{\prime}}=-0.8$ if CX84 is in the Galactic bulge. From VVV and UKIDSS data (Greiss et al. 2014; Lucas et al. 2008), the counterpart to CX84 has $J-K=1.41$ and $K_{S}=13.2$, yielding $M_{K}=-2$ and
$J-K=0.38$ at bulge distance after correcting for extinction. Wu et al. (2014) classify this object as a G9 star. Assuming a subgiant G9 star with $M_{r^{\prime}}=2.5$ as the donor based on the multiday suspected orbital period, and that reddening is roughly linear with distance, we derive a distance of $D \approx 3.4 \pm 0.7 \mathrm{kpc}$. At this distance, $L_{X} \approx 2.4 \times 10^{33} \mathrm{erg} \mathrm{s}^{-1}$ which is consistent with qLMXBs.

\section{9. $C X 87-D N$}

CX87 starts at close to $r^{\prime}=20.4$ at the beginning of our observations, and declines over the next few days to the limiting magnitude of our observations at $r^{\prime} \approx 23$ as shown in Figure 6 . 
This decline of 2-3 mag over a few days is consistent with a DN outburst. Measured from the magnitude when not in outburst, $\left(F_{X} / F_{\text {opt }}\right)=100$, which is too high for a non-magnetic CV. The faint magnitude in the optical and low density of stars in the field is consistent with some extinction. $E(B-V)=1.81$ in this field, making $\left(F_{X} / F_{\text {opt }}\right)=2$ at bulge distance, while $L_{X} \approx 1.3 \times 10^{33}(\mathrm{~d} / 8 \mathrm{kpc})^{2} \mathrm{erg} \mathrm{s}^{-1}$. This is brighter than is consistent with a non-magnetic $\mathrm{CV}$, suggesting it would have to be closer, which means it likely cannot suffer enough extinction to have $F_{X} / F_{\text {opt }}$ consistent with a non-magnetic $\mathrm{CV}$. DN have been observed in IPs, although rarely. It is possible that this is such a system at a distance of $2-3 \mathrm{kpc}$, which would give $L_{X}$ and $F_{X} / F_{\text {opt }}$ consistent with CVs. Because the X-ray observations and optical observations are not simultaneous, it is also possible that CX87 is a non-magnetic $\mathrm{CV}$ that was observed in the $\mathrm{X}$-ray at the start of the outburst, while the X-ray luminosity was still increases as a result of increased mass accretion rate and before quenching begins during DNe outbursts (Warner 2003). If the X-ray observations was taken during such an outburst, then $F_{X} / F_{\text {opt }}$ would be much closer to $1-10$, consistent with non-magnetic CVs undergoing DN outbursts. CX87 is classified spectroscopically by Torres et al. (2014) as a CV. Either scenario would make CX87 an interesting object for further study.

\subsection{CX161-Flare Star}

CX161 has a period of 3.32 days and shows a flare of 0.3 mag that fades back to the quiescent level before the next observation $2.3 \mathrm{hr}$ later, as shown in Figure 6. Absorbed $\left(F_{X} / F_{\text {opt }}\right)=1$, which could be consistent with a flaring $M$ dwarf between uncertainties in reddening, flux levels, and the fact that most of the bolometric luminosity for late $\mathrm{M}$ dwarfs is emitted in the IR rather than at optical wavelengths so that $F_{\text {opt }} \ll F_{\text {Bol }}$. The short timescale and magnitude of the flare is typical of flare stars, while the period is attributable to stellar rotation and star spots. The IR colors $H=13.885$ and $K_{S}=14.075$ from VVV data are also consistent with an M dwarf (Greiss et al. 2014). This object is suggested to be a chromospheric active star or binary after spectroscopic observations in Torres et al. (2014) pending further analysis, which agrees with our photometric classification.

\subsection{CX168-CV, qLMXB, or M Dwarf?}

CX168 shows variations with a possible period of 3.8 days as shown in Figure 6, but there is also some flickering superposed on these variations and a brightening of $0.1 \mathrm{mag}$ lasting at least a few hours on night 8 . Absorbed $\left(F_{X} / F_{\text {opt }}\right)=0.1$, which is consistent with CVs, qLMXBs, and active M dwarfs. The absolute magnitude of this object if it were in the Galactic bulge is $M_{r^{\prime}}=-6.1$ which too bright for CVs or qLMXBs, and would suggest a supergiant companion if this object were in the bulge rather than in the foreground. In addition, with bulge reddening, $\left(F_{X} / F_{\text {opt }}\right) \approx 10^{-4}$ which is too low for CVs or qLMXBs. Assuming a subgiant companion for a CV or qLMXB with an absolute magnitude of $M_{r^{\prime}}=2.5$ and that reddening is linear with distance, the colors and magnitudes are consistent with a distance of $2.3 \mathrm{kpc}$. Therefore, the true X-ray to optical flux ratio is likely toward the high end of the given range with the distance of the source substantially closer than the bulge. From 2MASS, this object has $J-K=1.14$, which is consistent with a nearby $M$ dwarf, though it could also be a qLMXB with a larger, cool companion at a greater distance. The small flare on night 8 of observations could be flaring in an active $M$ dwarf, while the absorbed $J-K$ values in 2MASS are consistent with an $\mathrm{M}$ dwarf in the foreground. In addition to the flare on night 8 , there is a small dip of 0.07 mag on night 6 that lasts less than $2 \mathrm{hr}$. Spectroscopy can quickly differentiate between an isolated $\mathrm{M}$ dwarf and an accreting $\mathrm{CV}$ or qLMXB with a subgiant donor.

\subsection{CX184-CV, qLMXB, or M Dwarf?}

CX184 has a photometric period of 0.811 days, or $19.5 \mathrm{hr}$. The unfolded light curve is shown in Figure 6, while the folded light curve is shown in Figure 7 . Absorbed $\left(F_{X} / F_{\text {opt }}\right)=1$, which is consistent with qLMXBs, CVs, or M dwarfs. The variations are single humped, with a steeper rise than decline, and have an amplitude of $0.1 \mathrm{mag}$. It is also possible that they are ellipsoidal variations with roughly equal minima, but the data are insufficient to differentiate the two. The higher X-ray to optical flux ratio in combination with a possible orbital period below a day makes this a candidate qLMXB or CV, though spectroscopic follow up is necessary to differentiate those two possibilities both from one another, which is non-trivial, and from a fast rotating active $\mathrm{M}$ dwarf. From VVV data, $J-K=0.74$, which could be consistent with either a foreground early $\mathrm{M}$ dwarf or a $\mathrm{CV}$ or qLMXB.

\subsection{CX251-M Dwarf, Possibly Binary}

CX251 is very steady except for a single dip at least 0.07 mag deep, shown in Figure 8, which could be part of an eclipse. The eclipse depth and duration are not well constrained as the last observation in night 2 is the only point in eclipse, so many different depths and durations are consistent with the data. Absorbed $\left(F_{X} / F_{\text {opt }}\right)=(1 / 10)$ which is consistent with qLMXBs, CVs, and M dwarfs, though the lack of ellipsoidal variations, even at high inclination, argues against a system accreting through Roche Lobe overflow. It is possible that this object is simply an eclipsing binary in the field coincident with the X-ray position. $F_{X} / F_{\text {opt }}$ is too high for the X-ray emission to be the result of coronal activity in this object unless it is an $M$ dwarf with most of the bolometric luminosity in the infrared. If the dip is part of an eclipse, the high $F_{X} / F_{\text {opt }}$ would suggest that both binary members are M dwarfs. $K=12.0$ and $J-K=1.63$ in VVV (Greiss et al. 2014), which is consistent with an M dwarf. This object is suggested to be a chromospheric active star or binary after spectroscopic observations in Torres et al. (2014) pending further analysis, which agrees with our photometric classification.

\subsection{CX279-CV or $q L M X B$}

We recovered no significant period for CX279 as determined by Monte Carlo simulations with the Lomb-Scargle statistic. This source appears to be eclipsing, shown in Figure 8, with eclipses of $0.6 \mathrm{mag}$ which are relatively broad, taking 4 out of 18 observations, implying they last for $22 \% \pm 11 \%$ of the orbital phase. Absorbed $\left(F_{X} / F_{\text {opt }}\right)=0.6$ which is consistent with qLMXBs and CVs. This object is also very faint in the infrared, at $K_{S} \approx 16.5$ (Greiss et al. 2014), which favors bluer sources such as CVs.

\subsection{CX291-Binary M Dwarfs or Interloper?}

CX291 has a single point in eclipse in the first night of observations, with a depth of $0.5 \mathrm{mag}$, shown in Figure 8. There is no variability apart from this eclipse; the weighted average as a model for the brightness has $\chi^{2} / \nu=1.4$ with the eclipse excluded. As with CX251, the only observation 

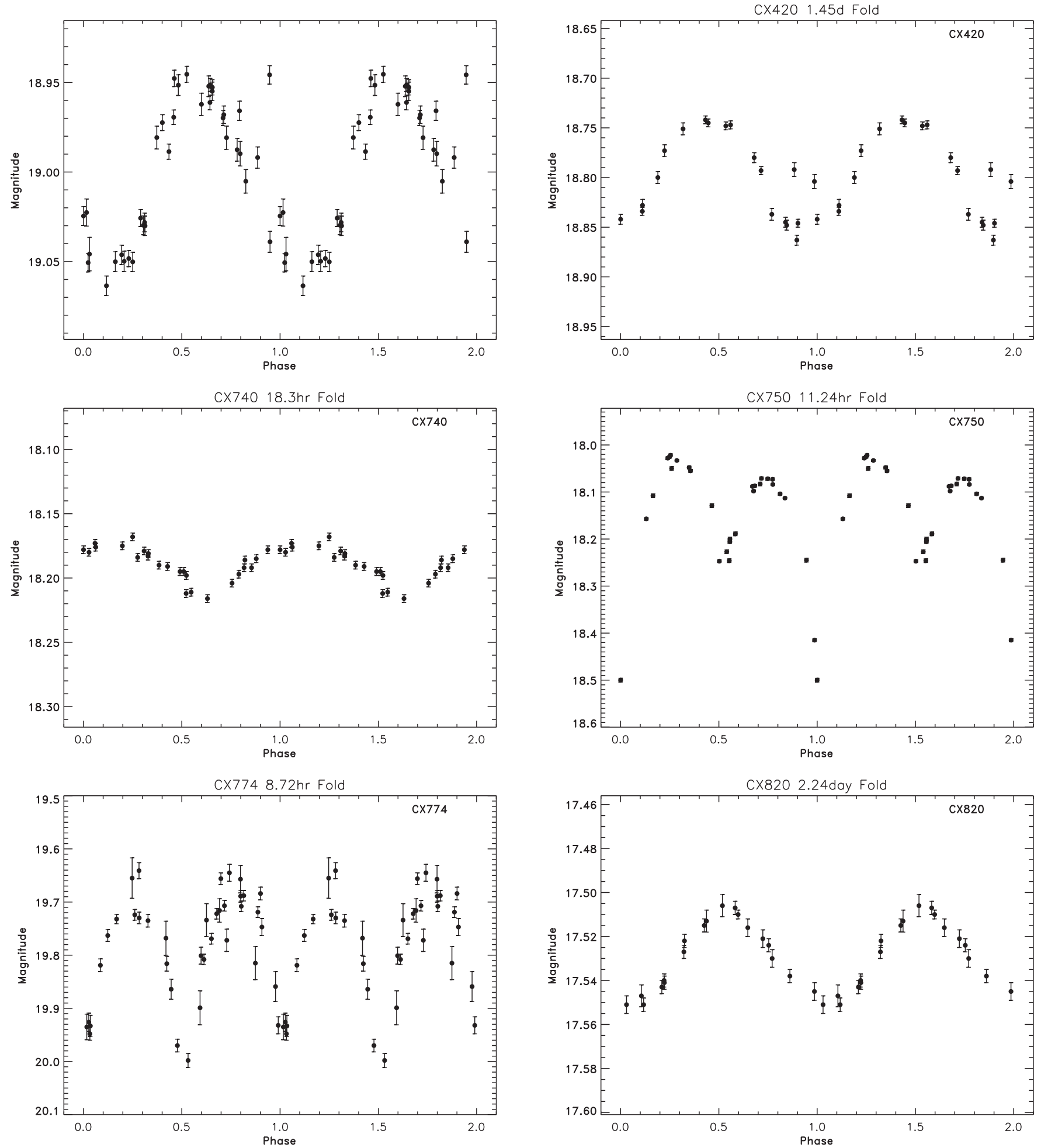

Figure 7. Top left: Mosaic-II light curve of CX184 folded on a $19.5 \mathrm{hr}$ orbital period. Top right: Mosaic-II light curve of CX420 folded on a 1.45 day orbital period. Middle left: Mosaic-II light curve of CX740 folded on an $18.3 \mathrm{hr}$ period. Middle right: light curve of one of the possible counterparts to CX750 folded on an $11.24 \mathrm{hr}$ period. Bottom left: light curve of one of the possible counterparts to CX774 folded on a $8.72 \mathrm{hr}$ period. Bottom right: light curve of CX820 folded on a 2.242 day period.

in eclipse comes at the end of the night, leaving its duration nearly unconstrained. The absorbed X-ray to optical flux ratio is 1 , which is consistent with CVs, qLMXBs, and M dwarfs, though the lack of ellipsoidal modulations from tidal distortion is problematic for a compact binary interpretation. Also, from VVV data (Greiss et al. 2014), $K_{s}=12.35$, which makes this object quite red. From 2MASS, $J-K=0.84$, which is also consistent with nearby $\mathrm{M}$ dwarfs, though $r^{\prime}-K_{S}=7.1$ could also point to significant reddening. Like CX251, this object could also simply be an interloping eclipsing binary.

\subsection{6. $C X 298-D N$}

CX298 drops from $r^{\prime}=21.4$ on the first night of observations to the limiting magnitude of $r^{\prime}=23$ by night 4 , where it remains 

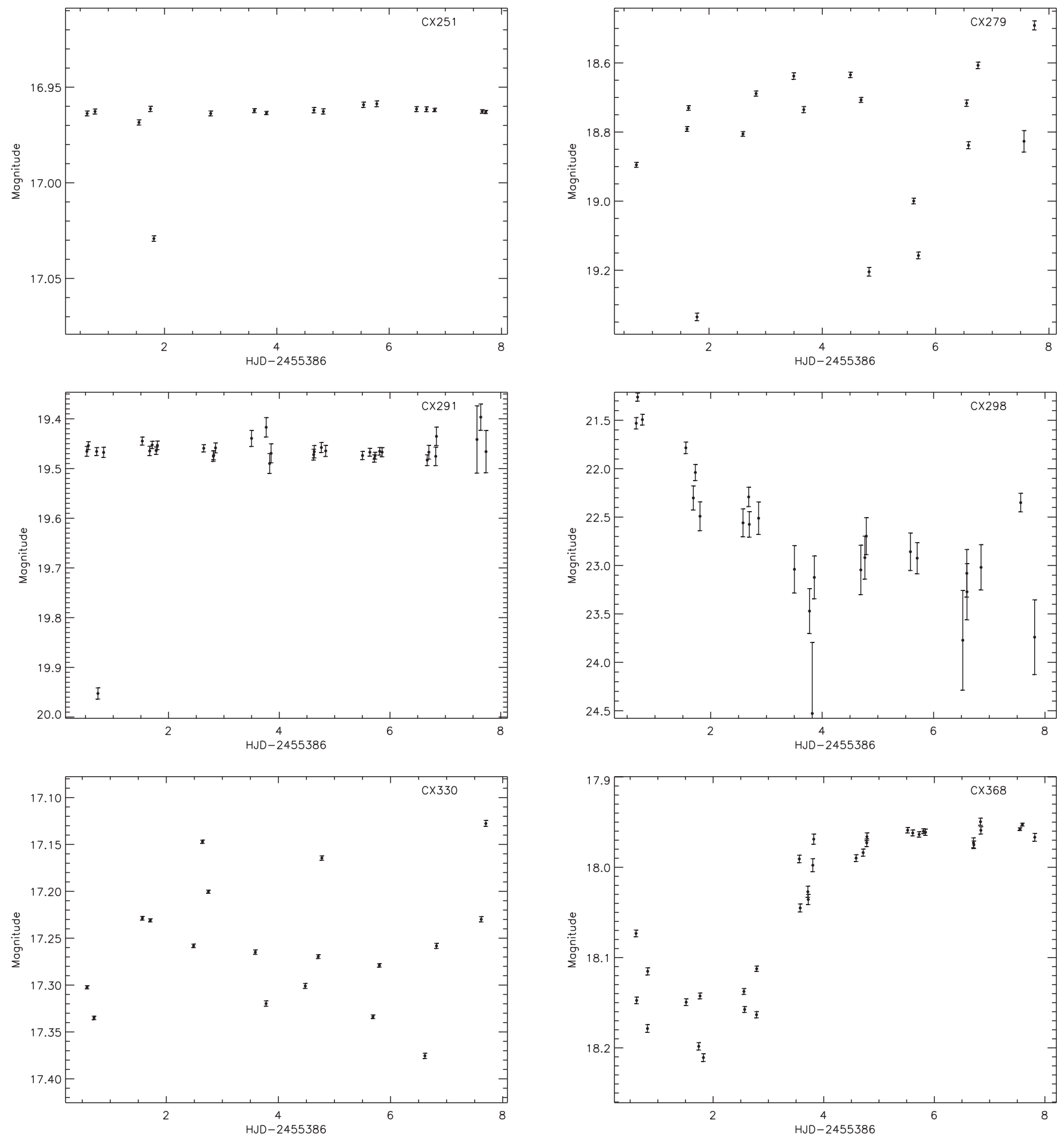

Figure 8. CX251, CX279, CX291, CX298, CX330, CX368 Mosaic-II light curves.

steady as shown in Figure 8. This large, steady decline could be the end of a DN outburst. The X-rays are likely emitted in quiescence, as the high accretion rate during the DN outburst quenches X-ray emission substantially (Collins \& Wheatley 2010; Patterson \& Raymond 1985). If the limiting magnitude is taken as the brightness in the quiescent state, absorbed $\left(F_{X} / F_{\text {opt }}\right)=20$, though it could be lower with reddening. At the bulge, $\left(F_{X} / F_{\text {opt }}\right)=1$, which is consistent with CVs. If it is a $\mathrm{CV}$ undergoing DN outbursts, however, then the distance should be substantially closer than the bulge as their X-ray luminosity is below what we are likely to detect at that distance. With a moderate amount of extinction, however, this ratio of X-ray to optical light is consistent with a CV undergoing DNe outbursts. This is a similar source to CX87, which also appears to be the end of a DN outburst with a very high X-ray to optical flux ratio, fading to or below our limiting magnitude. There is no infrared counterpart to this object in VVV or 2MASS, which further supports a DN interpretation.

\subsection{7. $C X 330$}

CX330 appears in OGLE-IV data (Udalski et al. 2012) as an irregular variable. In our data, it shows large amplitude aperiodic 
flickering covering a range of $0.3 \mathrm{mag}$ shown in Figure 8. The $\mathrm{X}$-ray to optical flux ratio is fairly low at 0.1 before correcting for extinction. The brightness varies on a timescale of hours, which makes it unlikely to be an AGN. The flickering is consistent with an accreting source.

\subsection{CX368-CV, qLMXB, or Symbiotic Binary?}

This source shows a dip of 0.2 mag before plateauing at the peak brightness, as shown in Figure 8 . The dip lasts for at least 3 days, though it begins before the start of our observations. The flickering present throughout the light curve favors an accreting binary instead of star spots on an M dwarf or RS CVn. The magnitude of the counterpart is $r^{\prime}=17.96$ which is consistent with a giant star at the bulge distance. This object appears in the 2MASS catalog at $J=14.185$ and $K=12.692$. For bulge reddening, $E(J-K)=0.91, A_{K}=0.63$. This implies $M_{K}=-2.46$ at the bulge which is also consistent with a giant star. In symbiotic binaries, X-rays are produced when winds from a giant star accrete onto a WD or NS. We have already identified a possible Carbon star symbiotic XRB in CX332 (Hynes et al. 2014), which are much more rare than ordinary symbiotics. Absorbed $\left(F_{X} / F_{\text {opt }}\right)=0.2$, which is consistent with CVs or qLMXBs, which cannot be ruled out. At bulge reddening, $\left(F_{X} / F_{\text {opt }}\right)=0.006$ which is also consistent with a symbiotic binary. $L_{X} \simeq 10^{32}(d / 8 \mathrm{kpc})^{2} \mathrm{erg} \mathrm{s}^{-1}$, which is also consistent with symbiotic X-ray binaries at the bulge.

\subsection{9. $C X 420-C V$ or $q L M X B$}

CX420 shows a period of 1.45 days. The Mosaic-II light curve is shown in Figure 9, while the folded light curve is displayed in Figure 7 . It has an absorbed $\left(F_{X} / F_{\text {opt }}\right)=0.4$ which is consistent with qLMXBs, CVs, and active M dwarfs. With the bulge distance reddening in this line of sight, $\left(F_{X} / F_{\text {opt }}\right)=0.002$ is consistent with coronally active stars as well, but those are too faint in the X-ray to be detected at bulge distance. This object is not very red, appearing in VVV data with $K_{S}=15.75$ and $J-K_{S}=0.63$, which rules out an active $\mathrm{M}$ dwarf. There is also some indication of flickering, which would also rule out an RS CVn or M dwarf. Long period qLMXBs or CVs indicate an evolved companion, which should have lower X-ray to optical flux ratios than those with MS donors due to the brighter donor. CX420 is consistent with such an object.

\subsection{0. $C X 426-D N$}

CX426 appears in OGLE-IV data (Udalski et al. 2012), where it is observed to undergo multiple DN outbursts. In our data, it shows a steady decline of 0.9 mag over the course of observations, shown in Figure 9, consistent with a DN outburst. The X-ray to optical flux ratio is 0.3 using the faintest observations from Mosaic-II data. This is consistent with a CV undergoing DNe outburst. There is some flickering around a best fit line as well with an amplitude 0.1-0.2 mag which is much higher than expected from simple photon noise or instrumental errors as well, which argues in favor of a CV interpretation as ordinary long period variables should not flicker. In VVV data (Greiss et al. 2014), this object is not firmly detected, though it can be seen by eye in some of the images. It is therefore quite blue, further supporting the interpretation of a $\mathrm{CV}$ undergoing DN outbursts. We independently strengthen the conclusion of Udalski et al. (2012), that this is a CV undergoing DN outbursts.

\subsection{CX437-CV or $q L M X B$}

CX437 shows two eclipses of at least a magnitude, shown in Figure 9. The counterpart is very faint, $r^{\prime} \approx 22.1$, and the eclipses drop below the limiting magnitude of our observations. There are 2 eclipses seen in 17 observations, suggesting an eclipse duration of $12 \% \pm 8 \%$ of the orbital phase, which is typical of CVs and qLMXBs. The eclipses last no more than $5 \mathrm{hr}$, which places an upper limit on the orbital period of 2 days. Absorbed $\left(F_{X} / F_{\text {opt }}\right)=10$ which is consistent with either qLMXBs or CVs with moderate extinction. Spectroscopic follow up is needed to differentiate between the $\mathrm{CV}$ and qLMXB interpretations, which will likely be possible because the inclination angle is strongly constrained by the fact that the system is eclipsing.

\subsection{2. $C X 476-D N$}

CX476 starts out near $r^{\prime}=21$ before dropping below the limiting magnitude after a few days, shown in Figure 9. This appears to be a CV undergoing DN outbursts. Absorbed $\left(F_{X} / F_{\text {opt }}\right)=20$ which is a little high for a $\mathrm{CV}$, but with some extinction and uncertainties in both $\mathrm{X}$-ray and optical fluxes, it is consistent. This object is not present in VVV (Greiss et al. 2014), which is unsurprising given its faintness in quiescence.

\subsection{3. $C X 645-C V$ or $q L M X B$}

CX645 shows large scale aperiodic variability, changing over a magnitude in brightness on a timescale of days as shown in Figure 9. One data point 1.7 mag below the nearest observation $5.5 \mathrm{hr}$ later appears to be an eclipse. There is also a large flare of $1 \mathrm{mag}$ in brightness that lasts no more than $3.7 \mathrm{hr}$ seen on the 8th night of observations. Absorbed $\left(F_{X} / F_{\text {opt }}\right)=(1 / 4)$, while with bulge distance reddening, unabsorbed $\left(F_{X} / F_{\text {opt }}\right)=0.003$. This range is consistent with a nearby $\mathrm{CV}$ or $\mathrm{qLMXB}$. The large amount of aperiodic variability suggests a larger contribution of continuum light from the disk rather than the donor star. This object does not appear in the VVV catalog (Greiss et al. 2014), meaning it is quite blue $\left(r^{\prime}-J \lesssim 2\right)$, which is consistent with continuum emission from an accretion disk. Spectroscopy is needed to further support any classification.

\subsection{CX705-Flare Star}

CX705 is in the OGLE-IV catalog (Udalski et al. 2012), which lists it as an irregular variable. It is also next to a Mira variable in OGLE-IV, which is at minimum light and much fainter than the counterpart to CX705 in our Mosaic-II data, though they are of comparable brightness in OGLE-IV I band photometry at the same time. Indeed, after examining the public OGLE-IV photometry of these two sources, it is evident that the counterpart to CX705 is being slightly contaminated by the light from the nearby Mira in OGLE-IV data, resulting in a slight rise in the average brightness of the counterpart to CX705 when the Mira variable is at maximum brightness. If these few observations are ignored, a strong period very near 1 day appears in the OGLE-IV data along with some flaring. In Mosaic-II data, CX705 has peaks in the periodogram at periods near 1 day and an integer fraction of 1 day, showing heavy aliasing between 1 day, $1 / 2$ of a day, and 1/3 of a day. None of these periods is significant in our data, though Monte Carlo simulations show that with any amount of flickering it is unlikely we would recover real modulations at such a period. This period and morphology from OGLE-IV data seems to shift very slightly over the length 

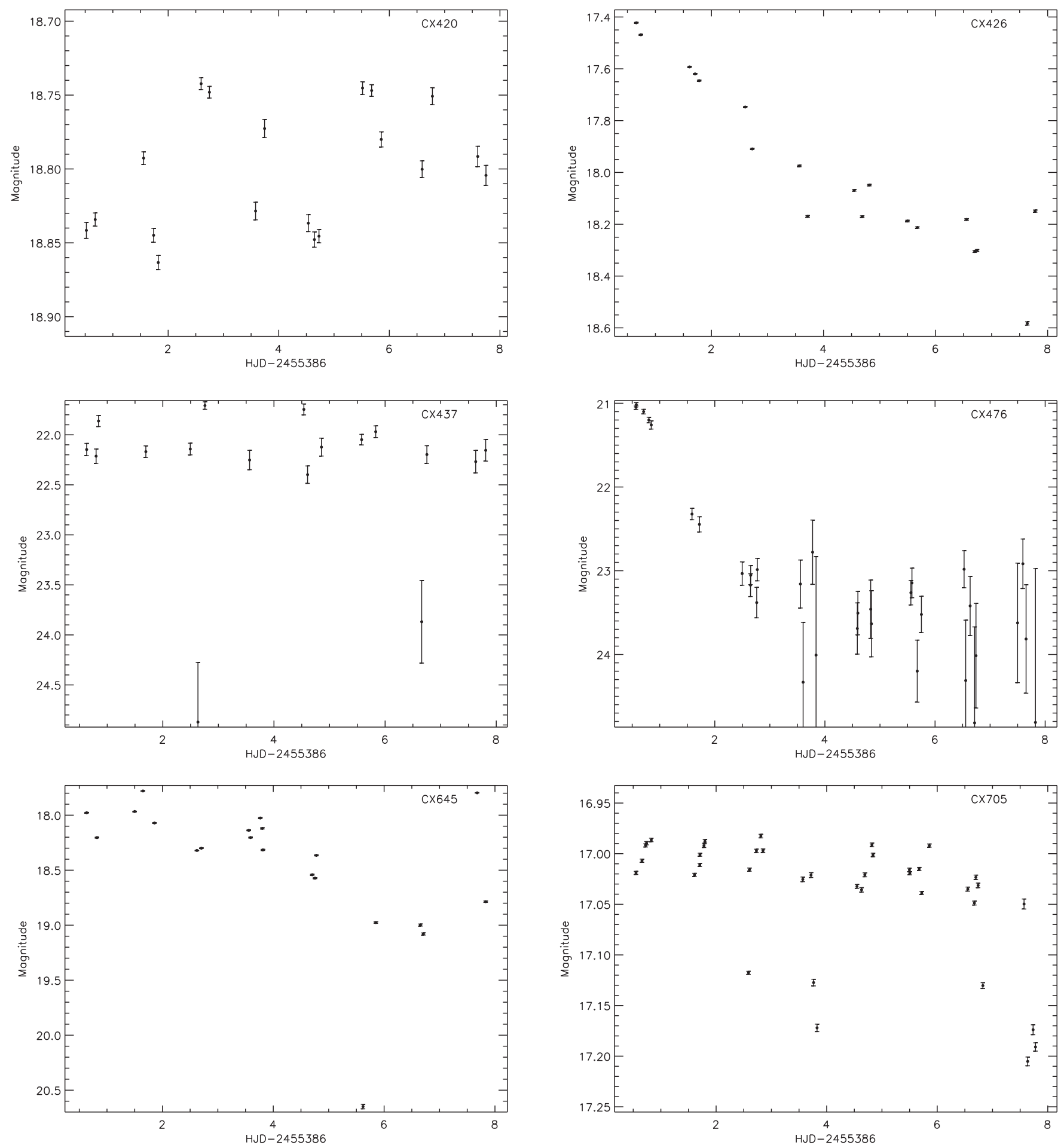

Figure 9. CX420, CX426, CX437, CX476, CX645, CX705 Mosaic-II light curves.

of the OGLE-IV observations, which could be attributable to star spots and differential rotation. CX705 is faint in the X-ray for its optical brightness, with absorbed $\left(F_{X} / F_{\text {opt }}\right)=0.05$, dropping to $1 / 1000$ at bulge reddening. This is consistent with coronal activity from a flare star, with some star spots appearing and disappearing over the course of OGLE-IV observations. This object appears in the VVV survey (Greiss et al. 2014) with $J-K_{s}=1.53$ and $K_{S}=12.48$. This is consistent with a flare star.

\subsection{5. $C X 740-C V, q L M X B$, or $M$ Dwarf}

CX740 shows modulations of $0.06 \mathrm{mag}$, shown in Figure 10, and with a period of 0.765 days, and is shown in Figure 7. Absorbed $\left(F_{X} / F_{\text {opt }}\right)=(1 / 6)$, though extinction in this line of sight is quite high and at bulge distance this drops to $2 \times 10^{-5}$. This range is consistent with qLMXBs, CVs, or active M dwarfs which have been observed to rotate this quickly, though the short period with the low X-ray to optical flux ratio for bulge 

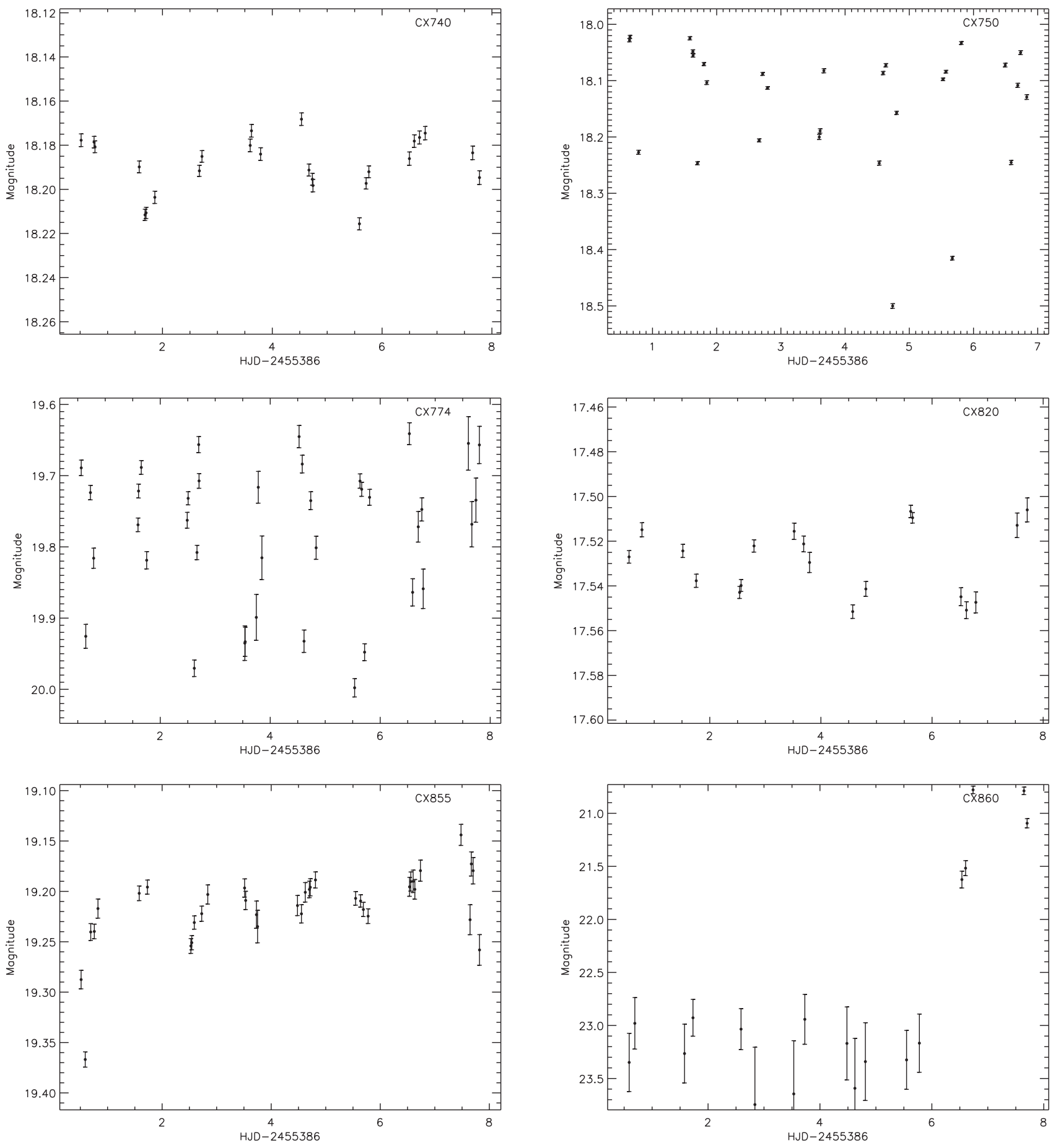

Figure 10. CX740, CX750, CX774, CX820, CX855, CX860 Mosaic-II light curves.

reddening strongly implies that this object is significantly closer than the bulge distance. The changes could also be ellipsoidal with a period of 1.53 days, but this is so close to $3 / 2$ of a day that we cannot distinguish between the periods because of gaps in phase coverage. The lower values of the X-ray to optical flux ratio are favored by larger distances, but it is unlikely that we would detect a W UMa system much further than several hundred parsecs because they are not luminous enough in the $\mathrm{X}$-ray to be detected at the bulge in our short X-ray exposures, and $\mathrm{W}$ UMas are unlikely to have a period as long as 1.5 days. CX740 is a candidate qLMXB or CV based on the possibility of ellipsoidal variations, but some active $M$ dwarfs have rotation periods below a day as well. From VVV data (Greiss et al.
2014), $J-K_{S}=1.18$ without correcting for reddening, which changes to $J-K_{S}=-0.88$ for a bulge distance reddening of $E(J-K)=2.06$. The color is consistent with a foreground $\mathrm{M}$ dwarf, and we cannot rule this possibility out either without spectroscopy, which should quickly differentiate between these cases.

\subsection{CX750—CV or qLMXB Plus a Field W UMa?}

There are two variable stars within the X-ray error circle for CX750. Both show what appear to be ellipsoidal variations, one also shows an eclipse. The Chandra observation that detected this source was made at a large off-axis angle, and the $95 \%$ confidence region has a radius of $11^{\prime \prime} .9$. The variable closest 
to the center of this region shows ellipsoidal modulations with $P_{\text {orb }}=1.12 \pm 0.03$ days and a mean magnitude of $r^{\prime}=19.4$. It is possibly a field W UMa, which would imply an X-ray brightness, given typical values for W UMa stars, of $\sim 10^{-15} \mathrm{erg} \mathrm{cm}^{-2} \mathrm{~s}^{-1}$ which is well below the X-ray detection limit. If it is the true counterpart to the X-ray source, it has an absorbed $\left(F_{X} / F_{\text {opt }}\right)=(1 / 20)$, which could be consistent with $\mathrm{CVs}$ or qLMXBs, while the period above a day would suggest a somewhat evolved donor star in either scenario if this were to be the true counterpart, though a MS donor could be possible for a $\mathrm{BH}$ qLMXB following the period-mass relation for MS donors in Frank et al. (2002). This object appears faintly in the VVV survey (Greiss et al. 2014) at $K_{S}=15.94$ and $J-K_{S}=0.59$ or $J-K_{S}=-1.17$ for bulge distance reddening, for which $\left(F_{X} / F_{\text {opt }}\right)=3 \times 10^{-5}$. This strongly implies that this object is closer than the bulge distance.

The other object has a period of $0.468 \pm 0.005$ days, or $11.24 \mathrm{hr}$, with a minimum $0.25 \mathrm{mag}$ deeper at phase $\phi=0$ than at phase $\phi=0.5$ (with the phase set arbitrarily to zero at the deeper minimum), which is consistent with qLMXBs and $\mathrm{CVs}$, as is the observed absorbed X-ray to optical flux ratio of $1 / 8$. The Mosaic-II light curve is shown in Figure 10, and the folded light curve is shown in Figure 7. The period is consistent with W UMas, but the asymmetry in the minima depths means that the temperatures of the two bodies are substantially different which is not the case for a W UMa in which the two stars share a common envelope. Indeed, the dip at $\phi=0.5$ is consistent with a minima from ellipsoidal variations without an eclipse, while the eclipse at $\phi=0$ is incompatible with ellipsoidal modulations, which means this is unlikely to be a W UMa. The maxima of the ellipsoidal modulations are also asymmetric, though the brighter maximum trails the deep eclipse in phase, while a hotspot from an accretion stream impact point on the disk should lead donor star in phase; this behavior is observed in the LMXB 4U 1822-37 (Mason et al. 1980) and the white dwarf CAL 87 (Schandl et al. 1997) which was also modeled as a spray of colder matter obscuring the inner disk region before the eclipse, causing the asymmetry. These objects, however, are intrinsically much more luminous than CX750 would be at bulge distance. This object appears in VVV data with $J-K_{S}=0.83$ and $K=13.89$. Because the period is only $11 \mathrm{hr}$, we can place a limit on the spectral type of the donor. Using the mass-period relation in Frank et al. (2002), this implies a donor mass of $\approx 1.2 M_{\odot}$ for a MS donor, which would mean this object has an intrinsic color of $J-K_{S}=0.24$ (Ducati et al. 2001). Assuming that reddening is linear with distance, $E(J-K) \approx 0.6$ implies $d=2.7 \mathrm{kpc}$ and $A_{r^{\prime}}=3.2$. This allows a tighter lower limit $\left(F_{X} / F_{\text {opt }}\right)>0.012$, as more evolved donors which fit in this period are redder and optically fainter, requiring less extinction to make the observed colors match. At a distance of $2.7 \mathrm{kpc}$, $L_{X}=4.3 \times 10^{31} \mathrm{erg} \mathrm{s}^{-1}$, which, given the orbital period, favors a quiescent $\mathrm{BH}$ accretor over a NS (Rea et al. 2011; Garcia et al. 2001). This object is a candidate eclipsing qLMXB, and spectroscopic follow-up is necessary to distinguish between $\mathrm{CV}$ and qLMXB interpretations.

\subsection{CX774-CV or qLMXB Plus an Interloper}

There are two variable stars within the X-ray error circle for CX774 as well. One undergoes a smooth decline of 0.04 mag over the 8 days of our observations, which is consistent with OGLE-IV observations showing a period of 43.478 days (Udalski et al. 2012). This object is very bright in the infrared in the 2MASS survey (Skrutskie et al. 2006), with $J-K=2.38$ and $K=7.60$, suggesting this object is heavily absorbed. For bulge distance and reddening for 2 MASS data, $M_{K}=-7.7$ and $J-K=1.28$. This suggests that this object is a red giant most of the way to the bulge, behind most of the dust in this line of sight. Absorbed $\left(F_{X} / F_{\text {opt }}\right)=10^{-2}$, while assuming reddening equivalent to bulge distance yields $\left(F_{X} / F_{\text {opt }}\right)=2 \times 10^{-4}$, and $L_{X}=4 \times 10^{32}(d / 8 \mathrm{kpc})^{2} \mathrm{erg} \mathrm{cm}^{-2} \mathrm{~s}^{-1}$. If this is the true counterpart, it could be a giant star in contact with a compact object, such that the variations observed are ellipsoidal modulations.

The other object shows ellipsoidal modulations with a period of 0.362 days, or $8.72 \mathrm{hr}$. The original light curve is shown in Figure 10, and the folded light curve is shown in Figure 7. The flickering in the light curve argues against this object being a W UMa. The variable showing ellipsoidal variations has a magnitude of $r^{\prime}=19.8$, which means it is likely below the $\mathrm{X}$-ray detection limit if it were a W UMa star. If it is the $\mathrm{X}$-ray source, absorbed $\left(F_{X} / F_{\text {opt }}\right)=0.6$, which is consistent with CVs and qLMXBs. The orbital period detected is also consistent with either interpretation. This object is swamped by the light from the IR-bright star discussed above in VVV data, and it is not in the 2MASS catalog. Assuming reddening equal to that at bulge distance, $\left(F_{X} / F_{\text {opt }}\right)=10^{-2}$, which is lower than typical values of NS qLMXBs, while a $\mathrm{CV}$ is unlikely to be as bright as $L_{X}=4 \times 10^{32} \mathrm{erg} \mathrm{cm}^{-2} \mathrm{~s}^{-1}$. A BH qLMXB with this orbital period is unlikely to remain at this luminosity for very long as well (Garcia et al. 2001; Kong et al. 2002; Lasota 2008; Rea et al. 2011; Jonker et al. 2012), so if this is the true counterpart, it is more likely a CV or qLMXB in the foreground rather than all the way to the bulge.

The X-ray source could realistically be either variable, but the one with the short period is certainly a close binary, and the flickering observed on top of the periodic changes is an indicator of accretion. If the short period variable is the X-ray source, then it could be a qLMXB or CV. We suspect that the second object discussed is the true counterpart, but spectroscopic follow up is necessary to fully determine which variable star is the true counterpart.

\subsection{8. $C X 820-R S C V n$}

CX820 shows smooth sinusoidal variations on a period of 2.242 days and an amplitude of only $0.02 \mathrm{mag}$, shown in Figure 10. The folded light curve is displayed in Figure 7. Absorbed $\left(F_{X} / F_{\text {opt }}\right)=0.1$ which is consistent with $\mathrm{RS}$ CVns, active M stars, qLMXBs, and CVs. The small amplitude of variation, lack of flickering, and multiday period together is suggestive of an RS CVn. The infrared colors of this possible counterpart are also consistent with an RS CVn (Greiss et al. 2014). This object is primarily of note in this work as a demonstration of how well the photometry methods can work even for very small amplitude changes. This object is suggested to be a chromospheric active star or binary after spectroscopic observations in Torres et al. (2014) pending further analysis, which agrees with our photometric classification.

\subsection{9. $C X 855-C V$ or $q L M X B$}

CX855, shown in Figure 10, has an orbital period of $1.82 \pm$ 0.05 days and a shallow, brief eclipse of at least $0.12 \mathrm{mag}$, though the depth is not well constrained. The rise and fall are asymmetric, so the light curve is not very well fit by a sine wave. The folded light curve is shown in Figure 11. All of the observations on night 8 are slightly higher than 

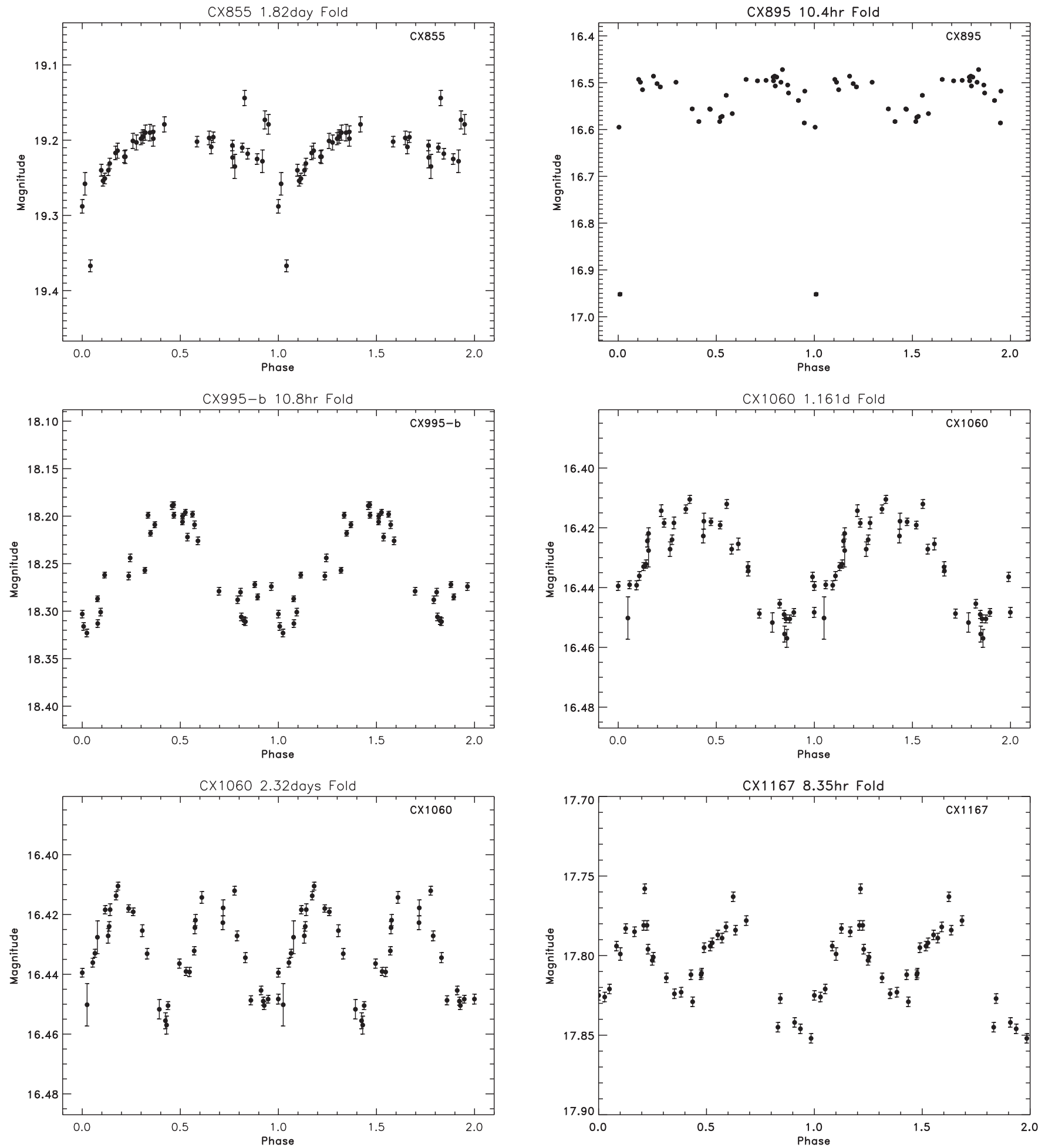

Figure 11. Top left: light curve of CX855 folded on a 1.8 day period. Top right: light curve of CX895 folded on a 10.4 hr period. Middle left: light curve of the second possible counterpart to CX995 folded on a 0.45 day period. Middle right: light curve of one possible counterpart to CX1060 folded on a 1.161 day period. Bottom left: the same possible counterpart of CX1060 plotted with twice the period. Bottom right: light curve of the likely counterpart to CX1167 folded on a 8.35 hr period.

other observations at the same phase, suggesting some intrinsic brightening of the source. Such level changes are commonly seen in accreting systems. Absorbed $\left(F_{X} / F_{\text {opt }}\right)=0.4$, which is consistent with qLMXBs and CVs. From VVV data, $J-K_{S}=$ 0.814 and $K_{S}=15.36$. The multiday orbital period implies an evolved donor for a Roche Lobe filling companion to the compact object. An absolute magnitude $M_{K}=2$, assuming that reddening is linear with distance, implies a distance of $\sim 4 \mathrm{kpc}$, and $\left(F_{X} / F_{\text {opt }}\right)=0.04$, which is also consistent with CVs and qLMXBs, with $L_{X}=2.6 \times 10^{32}\left(d / 8 \mathrm{kpc}^{2} \mathrm{erg} \mathrm{s}^{-1}\right.$. Phase-resolved spectroscopy is needed to determine the mass of the primary, which could be realistically either a WD, NS, or $\mathrm{BH}$ accretor, a task which is greatly simplified by the presence of eclipses which constrain $\sin i$. 


\subsection{0. $C X 860-D N$}

CX860 shows an outburst of at least 2.2 mag shown in Figure 10, from $r^{\prime}=23$ to at least $r^{\prime}=20.8$. The counterpart is rising in brightness on night 7 of observations and is falling again on night 8 . The outburst peaks during the day between these observations. An outburst lasting only 2-3 days is fast for a $\mathrm{DN}$, though it is not without precedent. The dramatic increase in luminosity is certainly consistent with a DN outburst. Absorbed $\left(F_{X} / F_{\text {opt }}\right)=10$, which is consistent with CVs undergoing DNe outburst, especially once reddening is taken into consideration. This object does not appear in VVV data (Greiss et al. 2014), which is expected for an object as intrinsically blue as a CV that is this faint in the optical. This appears to be a CV undergoing a DN outburst.

\subsection{CX895-Eclipsing CV, qLMXB, or Binary M Dwarfs}

CX895, shown in Figure 12, shows a possible period of $0.434 \pm 0.005$ days, or $10.4 \mathrm{hr}$, with a FAP of $1.2 \%$ with ellipsoidal modulations, shown in Figure 11. This period was found with phase dispersion minimization rather than the Lomb-Scargle statistic, and agrees with the period found in Udalski et al. (2012) of 0.42973 days. Udalski et al. (2012) also classify this object as eclipsing, which we confirm after observing an eclipse with a depth of at least 0.35 mag. There is some flickering in the light curve on the order of $0.03 \mathrm{mag}$, which argues strongly against this being a W UMa or RS $\mathrm{CVn}$. The asymmetry between minima also argues strongly against CX895 being a W UMa. This flickering is apparent in OGLE-IV data as well. This source is optically bright, near the nonlinear regime, and the relative photometric errors are generally much less than the observed dispersion. It is present in VVV photometry, with $K_{S}=13.73$ and $J-K_{S}=0.89$ (Greiss et al. 2014), which is consistent with an M dwarf, RS CVn or a compact binary where the continuum light is dominated by a cool companion. The ellipsoidal modulations indicate that if this is a compact binary, then the donor star is a significant contributor to the continuum light. Absorbed $\left(F_{X} / F_{\text {opt }}\right)=(1 / 40)$, which is consistent with CVs, M dwarfs, or qLMXBs. This object is suggested to be a chromospheric active star or binary in spectroscopic observations in Torres et al. (2014) pending further analysis or observation, which could be a result of having a cool donor that dominates the optical spectrum. There are M dwarf binaries known with shorter periods than CX895, and we cannot rule out this interpretation without further observation.

\subsection{2. $C X 957-C V$ or $q L M X B$}

CX957 shows no variability except for 3 points out of 36 dipping in what could be part of eclipses, shown in Figure 12. The eclipse is brief, lasting no more than $2 \mathrm{hr}$ as the observations before and after those in eclipse are back to the steady state. We can place an upper limit on the orbital period of 1 day, assuming that phases are evenly sampled. The eclipses are at least $0.13 \mathrm{mag}$ deep. This object is quite red, as it is near saturation in VVV $K_{S}$ band photometry (Greiss et al. 2014). It is in the 2MASS catalog with $J-K=1.83$, which becomes $J-K=0.81$ assuming reddening values at bulge distance. Absorbed $\left(F_{X} / F_{\text {opt }}\right)=0.2$ which is consistent with qLMXBs and $\mathrm{CVs}$, while at bulge reddening this drops to $\left(F_{X} / F_{\text {opt }}\right)=(1 / 100)$, which favors CVs and BH qLMXBs, but those are likely too X-ray faint at allowed periods to be detected at bulge distance. This is likely a compact binary in quiescence with a very cool donor.

\subsection{3. $C X 973-M$ Dwarf, $C V$, or $q L M X B$}

CX973 appears in OGLE-IV data (Udalski et al. 2012) with a period of 10.352 days. Our Mosaic-II data are consistent with this, showing smooth variations over several days shown in Figure 12 with a period of roughly $\sim 11$ days. The first hump is $0.1 \mathrm{mag}$ in amplitude, while the second is 0.15 mag above that. We observe only one minimum. Absorbed $\left(F_{X} / F_{\text {opt }}\right)=0.2$ which is consistent with qLMXBs and CVs or active $\mathrm{M}$ dwarfs, while at bulge reddening, $\left(F_{X} / F_{\text {opt }}\right)=0.002$ and $L_{X}=2 \times 10^{32}(d / 8 \mathrm{kpc})^{2} \mathrm{erg} \mathrm{s}^{-1}$. The possible long period and low X-ray to optical flux ratio at the bulge could be indicative of an evolved companion to an X-ray binary or CV. At the bulge, $M_{r^{\prime}}=-1.7$ which is consistent with a giant star. It is also bright in the infrared, with $K_{S}=12.79$ in VVV data (Greiss et al. 2014). The X-ray brightness is too high for coronal activity at bulge distance, while $F_{X} / F_{\text {opt }}$ is too high for coronal activity at nearer distances except for $\mathrm{M}$ dwarfs which emit most of their radiation in the IR. This could be a CV or qLMXB with an evolved donor or a nearby $M$ dwarf with a 10.352 day rotation period. Spectroscopy can quickly differentiate between the case of a cool spotted star or accreting compact binary.

$$
\text { 4.34. CX982-DN? }
$$

The light curve morphology of CX982 is unique. There is an outburst of $>1.3$ mag starting on night 6 of observations, but on night 7 it drops 2 mag from the peak of the outburst within $1.5 \mathrm{hr}$. It returns to its peak brightness at the next observation 36 minutes later. The full light curve is shown in Figure 12. There is nothing wrong with the images showing it back to or below its quiescent brightness, and there are three observations of it at low level within the apparent outburst. If it were not for these three points, it would appear to be a fairly typical DN outburst. It is possible that this is a high inclination system and the donor star blocks the rapidly accreting disk in these points, which would explain the sudden drops and increases in observed luminosity. It does not appear in VVV (Greiss et al. 2014), which is consistent with an intrinsically blue object of this magnitude such as a CV undergoing DNe outbursts.

\subsection{CX995-CV or qLMXB Plus Interloper}

CX995 also has two variables inside the X-ray confidence region. The reference image and variance image are shown in Figure 13. The fainter star of the two shows an eclipse $\sim 1$ mag deep, with no significant period, shown in Figure 12. The eclipse lasts over two observations separated by 20 minutes. The eclipse comes at the start of the night's observations, so there is no way to be sure exactly how deep the eclipse is or how long it lasts. A lower limit for the eclipse duration can be estimated if we assume that we see the eclipse at its full depth; it should last $1.3 \mathrm{hr}$. It is also possible that the first data point is taken as the source goes into eclipse, in which case the eclipse could be substantially shorter.

The second variable in the X-ray confidence region has a period of either 0.4517 days or 0.9034 days, depending upon whether the variations are ellipsoidal or sinusoidal. The 0.4517 day fold is shown in Figure 11. Our observations cannot adequately distinguish between the two in this case. Some 

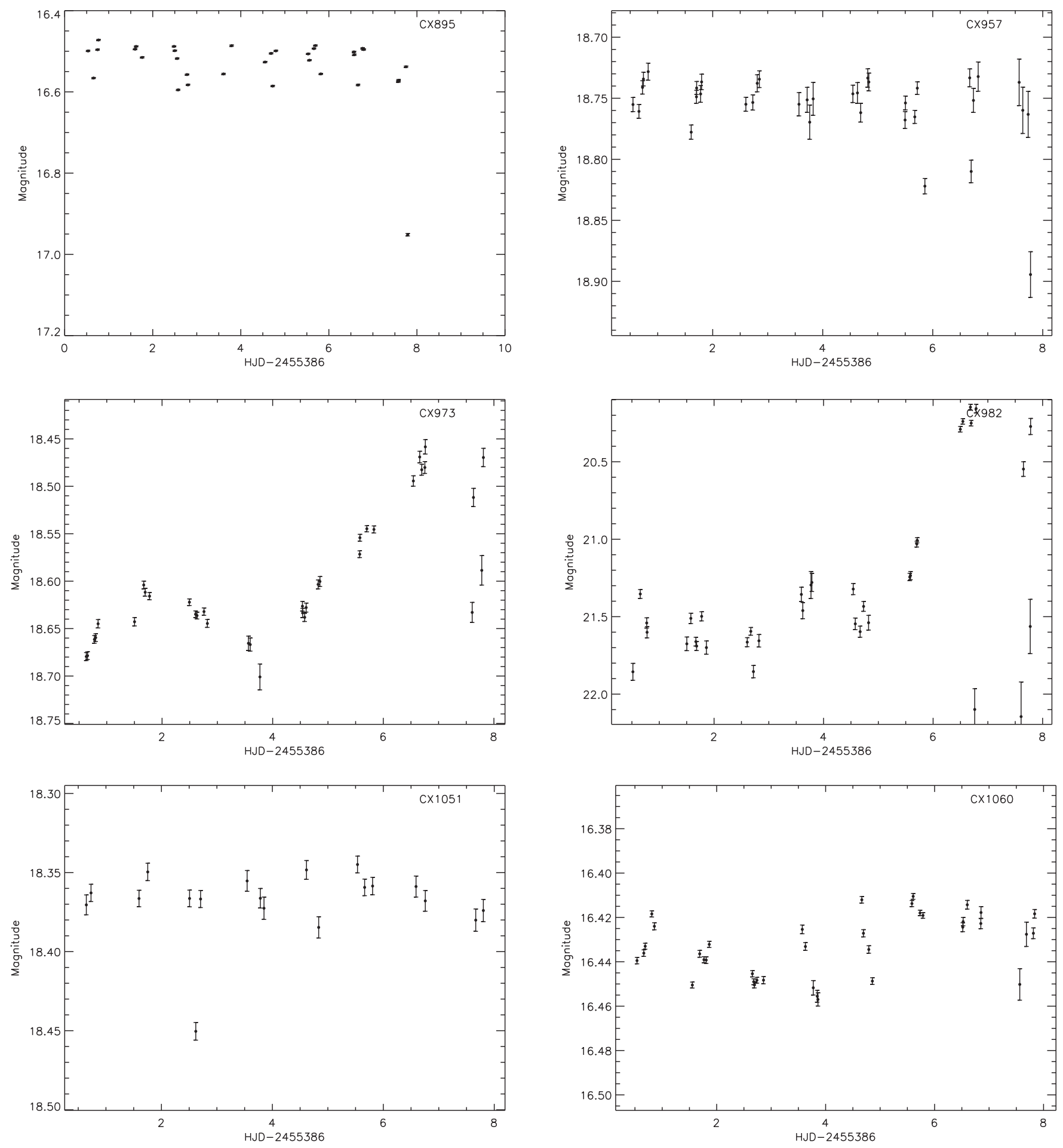

Figure 12. CX895, CX957, CX973, CX982, CX1051, CX1060 Mosaic-II light curves.

M dwarfs have rotation periods that fast. The second variable is marginally more likely to be the counterpart based solely on the proximity to the center of the X-ray position, while the first shows flickering and a deep eclipse which makes it the more likely candidate.

These two objects are blended in VVV data, with a color of $J-K_{S}=1.38$ and $K_{S}=13.54$ (Greiss et al. 2014), suggesting that at least one of these objects is fairly red. The flickering and eclipse in the fainter variable star are more easily explained as a compact binary than a field star, while the brighter second variable could be responsible for the red color in VVV as a spotted star in the field.

The eclipsing possible counterpart would have an absorbed $\mathrm{X}$-ray to optical flux ratio of 0.5 , which is consistent with non-magnetic CVs, qLMXBs, and magnetic CVs. The second variable would have an absorbed flux ratio of 0.1 , which is also consistent with $\mathrm{CVs}$, qLMXBs, and active $\mathrm{M}$ dwarfs. Spectroscopy is needed to distinguish between possibilities, as well as to confirm which of these two variables is the true counterpart to the X-ray source. 

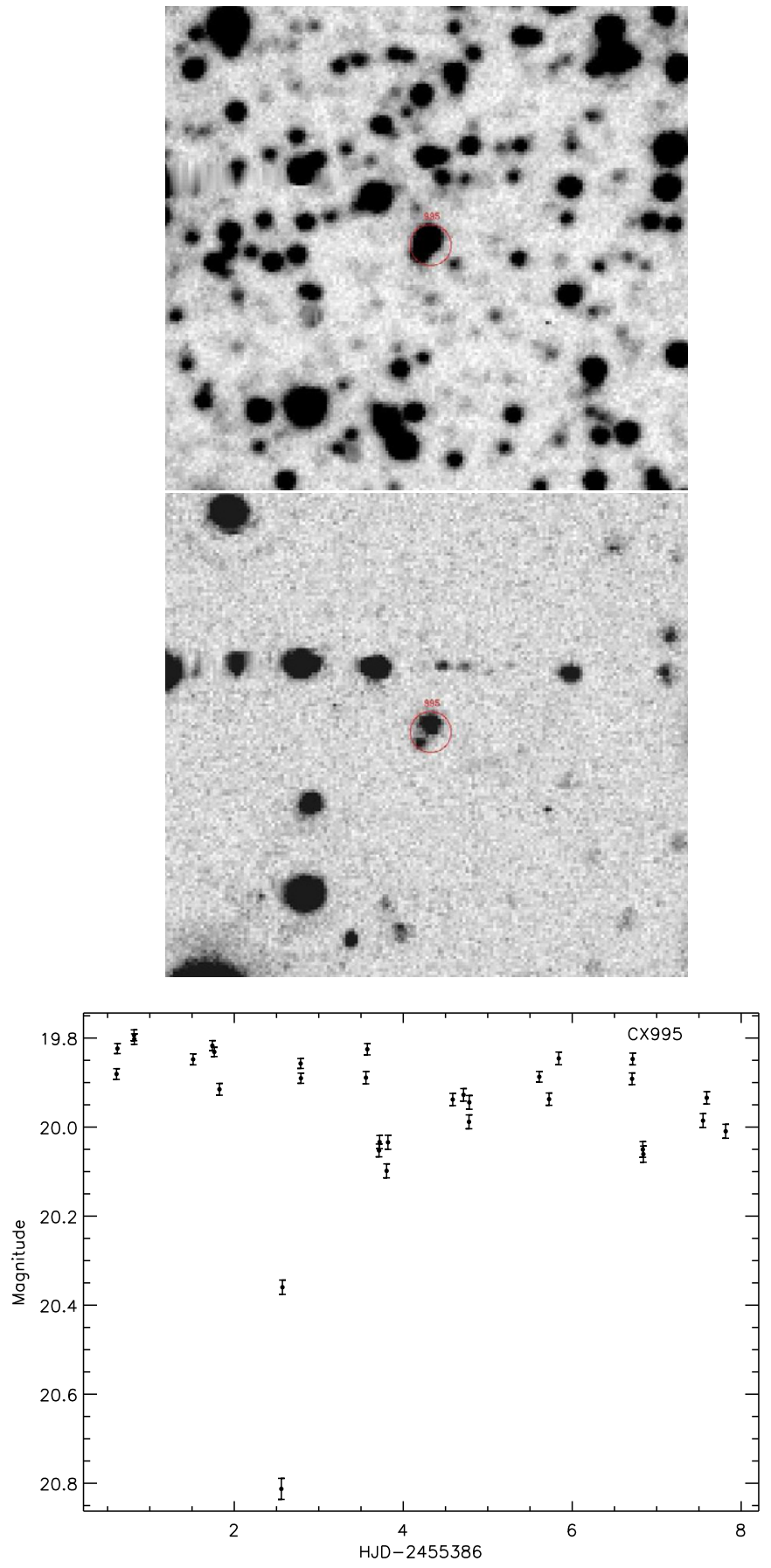

Figure 13. Top: finder chart for CX995 with X-ray position plotted in red. Middle: variance map of Mosaic-II images of this field. Bottom: light curve of the eclipsing variable further from the center of the X-ray position for CX995, judged to be the more likely counterpart because of flickering and a short eclipse. Similar figures for each source with a variable star coincident with the X-ray position are available in the online edition of this article.

(The complete figure set (495 images) and a color version are available in the online journal).

\subsection{CX1051-CV or $q L M X B$ ?}

CX1051 has a dip that could be the ingress or egress of a brief eclipse at least $0.1 \mathrm{mag}$ deep, lasting no more than $2 \mathrm{hr}$, shown in Figure 12. Only 1 observation out of 19 is in eclipse. If we assume that we evenly sample all phases, and that the eclipse lasts the full $2 \mathrm{hr}$ possible and lasts only the $5 \%$ of the orbit indicated by seeing $1 / 19$ observations in eclipse, this places an upper limit of $40 \mathrm{hr}$ on the orbital period. Absorbed $\left(F_{X} / F_{\text {opt }}\right)=(1 / 7)$, which is consistent with qLMXBs and CVs.

\subsection{CX1060—CV or $q L M X B, R S C V n$ ?}

CX1060, shown in Figure 14, has periodic modulations with a fundamental period of 1.161 days, and an amplitude of only 0.05 mag. There is more scatter around the peak on this period than in the rest of the light curve. Doubling the period to 2.322 days does not produce a significantly better fit. Each fold is shown in Figure 11. Absorbed $\left(F_{X} / F_{\text {opt }}\right)=0.02$ which is consistent with an RS CVn, CV, or qLMXB, and drops further to $\left(F_{X} / F_{\mathrm{opt}}\right)=0.001$ assuming reddening at bulge distance, indicating that this source is more likely in the foreground as the types of objects found at this period and that low X-ray to optical flux ratio are not luminous enough in the $\mathrm{X}$-ray to be seen at the bulge in our survey. The dispersion above what expected from statistical noise is inconsistent with an RS CVn which should not show flickering. This object is therefore a candidate $\mathrm{CV}$ or qLMXB. In the qLMXB interpretation, the low value of $F_{X} / F_{\text {opt }}$ even without extinction favors a BH primary. This object is blended with a nearby star separated by 1 1.5 in VVV photometry with $K_{S}=10.0$ (Greiss et al. 2014). This IR-bright star is also within the $\mathrm{X}$-ray confidence region, but does not show significant variability in Sloan $r^{\prime}$ band photometry. The variable star itself does not appear in VVV photometry. Spectroscopy is needed to differentiate between cases and to reject more firmly an RS CVn interpretation.

\subsection{CX1086-CV, qLMXB, or M Dwarf}

CX1086 shows what appears to be an eclipse with a depth of 0.26 mag and FWHM duration of 2 days, shown in Figure 14. In OGLE-IV data this object exhibits the same behavior on a period of $P=11.768$ days (Udalski et al. 2012). In OGLE data, it is listed as an eclipsing source, with a minimum at phase $\phi=0.5$ that is not as deep as that at $\phi=0.0$ by $\Delta I=0.05$ mag. The OGLE light curve is also consistent with ellipsoidal variations. OGLE-IV observations span a period of $2 \mathrm{yr}$, and the lack of changes in the light curve morphology cuts weakly against the idea that the modulations are produced by starspots, which could produce changes in phase and amplitude, and even in period, in that time as star spots appear, disappear, and move toward the equator from the poles. Absorbed $\left(F_{X} / F_{\text {opt }}\right)=(1 / 6)$, which is consistent with CVs, qLMXBs, or M dwarfs but is too high for an RS CVn star, which is unlikely to be detected at sufficient distance to suffer the amount of extinction needed to lower $F_{X} / F_{\text {opt }}$ to a point consistent with RS CVns. An eclipse this broad or ellipsoidal modulations lasting this long would imply an evolved donor. At the bulge, $E(B-V)=2.11$ in this line of sight, which would imply $M_{r^{\prime}}=-1.77$, consistent with giant stars. $\left(F_{X} / F_{\text {opt }}\right)=0.002$ at the bulge, which is also consistent with qLMXBs with giant donors. In VVV data, this object is also variable and quite red at $K_{S}=12.20$ and $J-K_{S}=1.52$. Correcting for reddening assuming bulge distance, $J-K_{S}=0.39$ and $K_{S}=-3$, which is too blue and too bright for an red giant branch star, but would be consistent for a distance somewhat before the bulge, experiencing less reddening than the red clump stars used to estimate reddening in the VVV survey. This object is consistent with a qLMXB with an evolved companion, though spectroscopy is needed to completely rule out the scenario of a nearby coronally active M dwarf with a pattern of star spots which emulates either ellipsoidal modulations or a broad eclipse. 

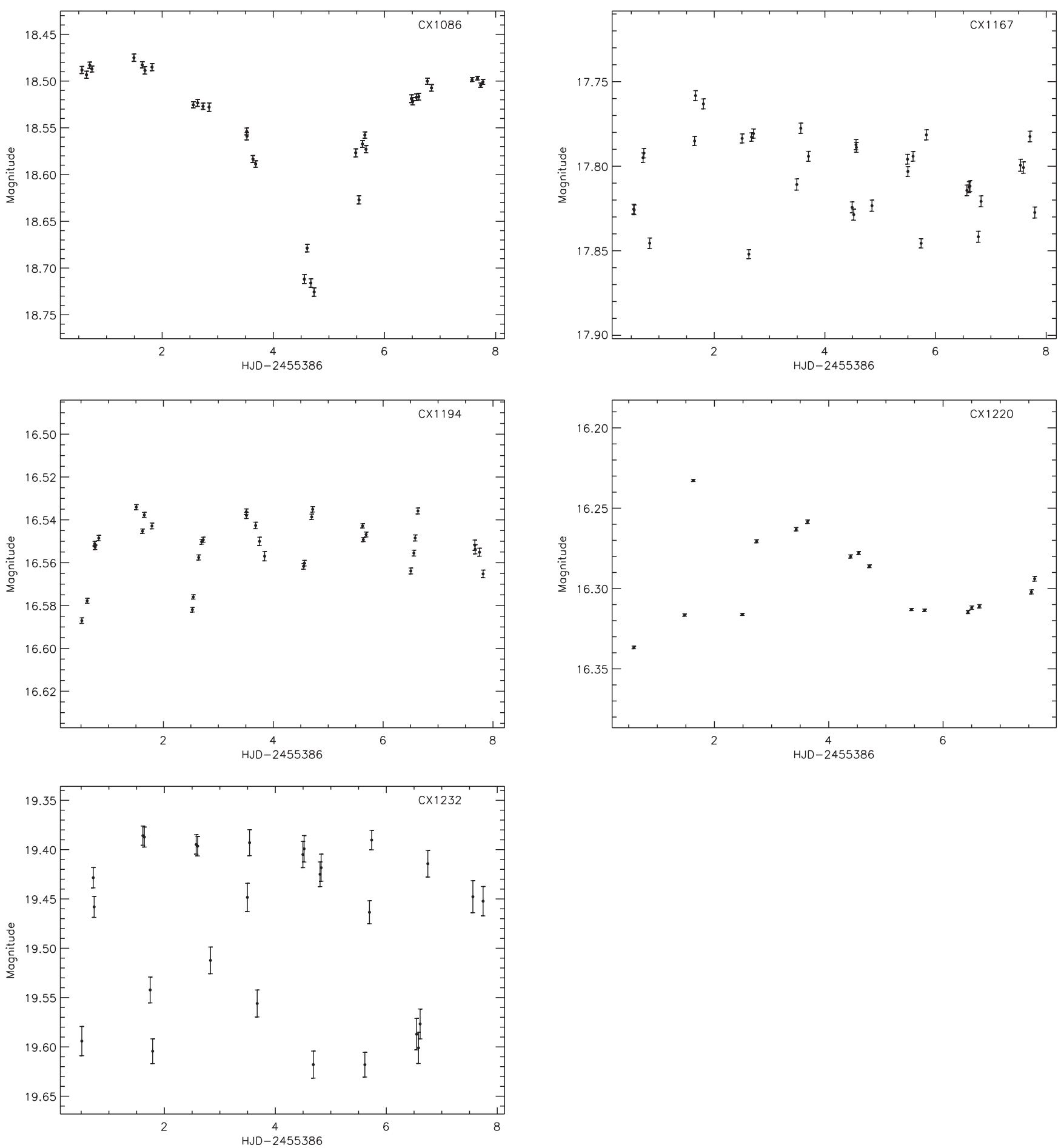

Figure 14. CX1086, CX1167, CX1194, CX1220, CX1232 Mosaic-II light curves.

\subsection{CX1167-CV or $q L M X B$}

CX1167 is one of the few X-ray sources with a counterpart showing ellipsoidal modulations at a period one expects for a qLMXB. The light curve is shown in Figure 14 and is shown in Figure 11 folded on $P_{\text {orb }}=8.35 \mathrm{hr}$. It has absorbed $\left(F_{X} / F_{\text {opt }}\right)=0.08$, which drops to 0.002 for bulge reddening. We would not see an object such as a W UMa or other low luminosity $\mathrm{X}$-ray emitter at the bulge, while reddening equivalent to being at the bulge distance is needed to make the observed quantities consistent with a W UMa. The low X-ray to optical flux ratio at bulge distance is also unlikely for CVs or qLMXBs, which favors the interpretation that CX1167 is closer than bulge distance, with the X-ray to optical flux ratio toward the higher end of the possible range. In VVV photometry for this object (Greiss et al. 2014), $K_{S}=14.4$ and $J-K_{S}=1.02$. This is consistent with a qLMXB or CV with a cool MS donor and moderate reddening.

\subsection{0. $C X 1194$}

CX1194, shown in Figure 14, has a significant period of 1.94 days, which is of note because it is so close to an integer of 

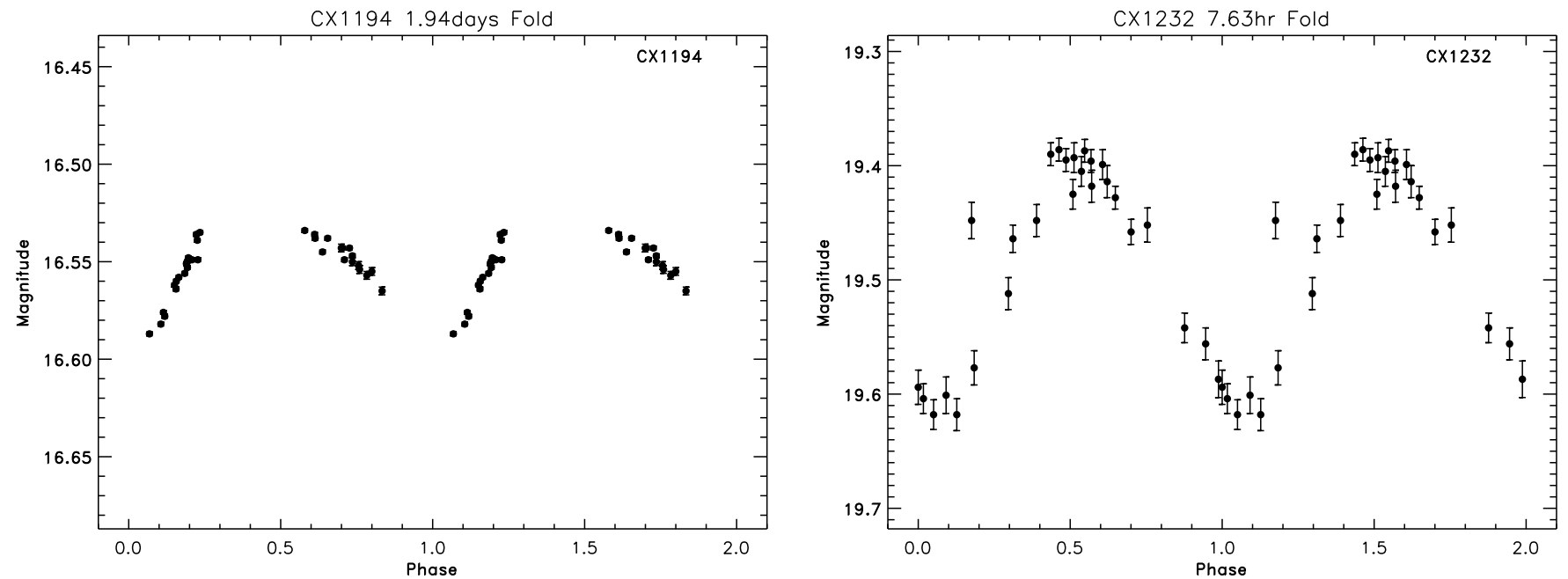

Figure 15. Left: light curve of one possible counterpart to CX1194 folded on a 1.94 day period. Right: light curve of the likely counterpart to CX1232 folded on a $7.63 \mathrm{hr}$ period.

1 day, and is strongly aliased by our nightly sampling rate. This aliasing is apparent in the folded light curve in the form of large gaps at near $\phi=0.4$ and $\phi=0.9$ seen in Figure 15. The gaps in phase coverage make it difficult to determine the exact amplitude of the variations, but they are on the order of $0.06 \mathrm{mag}$. The light curve is asymmetric and single-humped, rising faster that it falls. In VVV data, $K_{S}=14.19$ and $J-K_{S}=0.73$ (Greiss et al. 2014). Absorbed $\left(F_{X} / F_{\text {opt }}\right)=(1 / 40)$, which is consistent with RS CVns, CVs, or qLMXBs. If this object is a $\mathrm{CV}$ or qLMXB, the period would suggest a somewhat evolved donor star. Spectroscopy is necessary to firmly distinguish between possibilities.

\subsection{CX1220_Flaring M Dwarf or RS CVn}

CX1220 has a counterpart that is very near the nonlinear regime of the CCD response at $r^{\prime}=16.29$, and some observations in good seeing conditions were removed for being in this regime. This source shows a brief outburst of 0.1 mag and a rise and decline of $0.06 \mathrm{mag}$ over several days, shown in Figure 14. This could be a flaring $M$ dwarf or an RS CVn. Absorbed $\left(F_{X} / F_{\text {opt }}\right)=(1 / 50)$ which is consistent with RS CVns and active stars. This object appears in the 2MASS catalog (Skrutskie et al. 2006) and is quite red with $J-K=1.347$ and $K=11.311$. The very red color and IR brightness of this object, as well as the apparent flare, is consistent with a nearby flare star, but spectroscopy is necessary to firmly classify this object.

\subsection{2. $C X 1228$}

There is a variable star outside the $95 \%$ X-ray confidence region that is listed as a possible counterpart in Udalski et al. (2012). We include it in Table 2 to show that we confirm their results, but based on our selection criteria, it should not be considered as a likely counterpart to the X-ray source without other evidence, such as from spectroscopy.

\subsection{3. $C X 1232-C V$ or $q L M X B$}

CX1232 has a possible counterpart which is variable, but near the edge of the X-ray $95 \%$ confidence region. The light curve, shown in Figure 14, has a significant period of 0.3179 days, or $7.63 \mathrm{hr}$, with an amplitude of $0.24 \mathrm{mag}$. The true period could be twice this if the modulations are ellipsoidal, though the fit provided by doubling the period is no better. The folded light curve is shown in Figure 15. Absorbed $\left(F_{X} / F_{\text {opt }}\right)=0.4$ which is consistent with CVs and qLMXBs. The period, amplitude of oscillations, and X-ray to optical flux ratio are certainly suggestive of a compact binary, but spectroscopy is needed to classify this object securely.

\section{PERIOD DISTRIBUTION OF OPTICAL COUNTERPARTS}

Our observations have a high rate of period recovery for systems with little to no flickering. As aperiodic fluctuations begin to overtake the periodic, however, we lose the ability to reliably recover periods with the limited sampling we have. To quantify this, we have run Monte Carlo simulations to determine the rate of period recovery for various periods with our average level of sampling at different relative amounts of flickering, the results of which are shown in Figure 16. For qLMXB systems in which $\gtrsim 1 / 2$ of the light should come from the donor star rather than the accretion disk, we expect to recover almost all periods between 2 and $23 \mathrm{hr}$. We do suffer from aliasing around 1 day, which is an expected and unavoidable result of being able to observe only at night when only a single observatory is used. We cannot claim strong evidence of periods longer than 4 days because our baseline of observations only extends 8 days and we cannot, therefore, see a suspected period $>4$ days repeat. All periods listed greater than 4 days should be treated as suspect pending other observations. Some systems show a long rise or a single crest or trough. After matching these to the recovered counterparts in OGLE IV data (Udalski et al. 2012), the majority of these systems are long period variables with $P_{\text {orb }}>8$ days. Because larger stars will have longer periodicities, the shallower OGLE IV survey likely contains the majority of the long period objects in its field of view. Some of these objects are also irregular variables with characteristic timescales of days or longer, such as CX332 (Hynes et al. 2014).

The period distribution, shown in Figure 17, is a broad indicator of the types of sources we are finding. RS CVns tend to have periods of days, W UMas less than a day, while the period of LMXBs depends on the mass and evolutionary stage of the donor. The naive expectation from Kepler's Third Law is that the mass of the primary will affect the period of the system, 


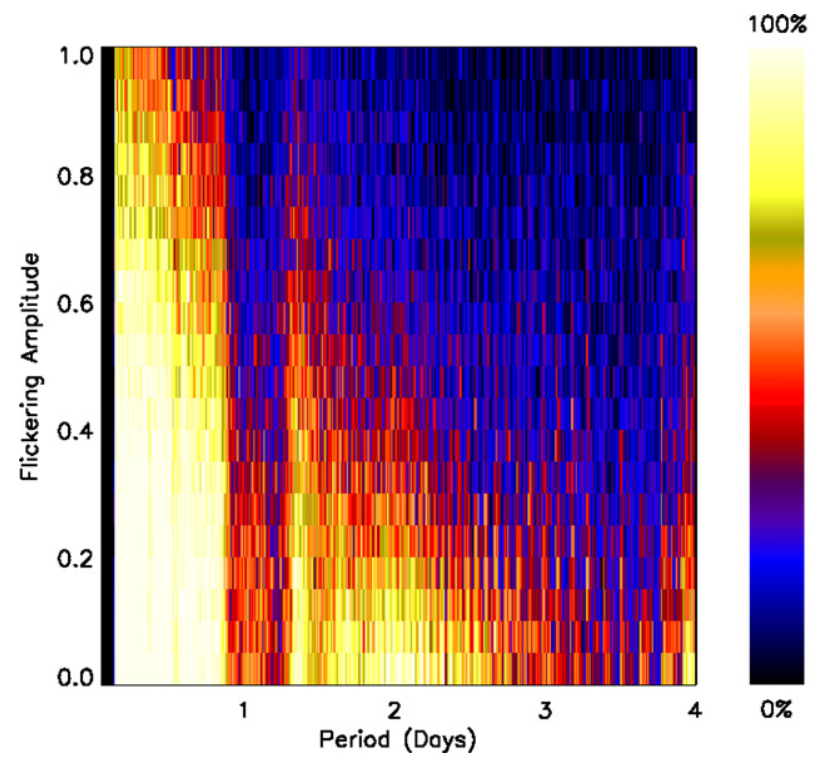

Figure 16. This plot shows the likelihood of recovering a given period at different levels of flickering imposed on the underlying periodicity for our Mosaic-II observations. As you can see, it is unlikely that we recover photometric periods below $2 \mathrm{hr}$, though we maintain better than even chances of recovering periods between $2 \mathrm{hr}$ and 1 day through the rms of random variations being $80 \%$ of the amplitude. Loss of sensitivity due to aliasing around 1 day is apparent. For qLMXBs with periods above 1 day, the donor should comprise a larger fraction of the continuum light because of its larger radius, so random flickering originating in the disk should be smaller amplitude compared to shorter period systems. For RS CVns and W UMas, there should be no intrinsic flickering, with the only noise being photometric error on the order of $<1 \%$ for sources brighter than $r^{\prime}=19$.

(A color version of this figure is available in the online journal.)

but the Roche Lobe geometry changes as a function of the mass ratio in such a way as to counter this dependence. For MS donors, the period is on the scale of hours with $M_{2} \approx 0.11 P_{\mathrm{hr}}$ (Frank et al. 2002), but periods of days are possible for more evolved donors. Of the population of currently known LMXBs, the period distribition peaks around 5-6 hr (Lewin \& van der Klis 2006), though systems with $P_{\text {orb }}>1$ day are certainly known. It is also important to note that the current selection effects in finding LMXBs in outburst and following them into quiescence select against short period systems (Knevitt et al. 2014). Non-magnetic CV systems, of which we expect $\approx 46$ with optical counterparts in the Mosaic-II area, have a bimodal orbital period distribution peaking at $1.5 \mathrm{hr}$ and $3.5 \mathrm{hr}$ for X-ray selected CVs, with a substantial gap in between 2-3 hr that is known as the CV period gap (Gänsicke 2005; Warner 1976). We are unable to reliably recover with confidence periods below the CV period gap. We expect hundreds of RS CVn systems (Jonker et al. 2011). The population assumptions used in Jonker et al. (2011), converted into Sloan $r^{\prime}$ for this work, predict only 18 qLMXBs with $r^{\prime} \leqslant 21.5$ that we are likely to detect, so the vast majority of systems with $P_{\text {orb }}>1$ day should be RS CVns rather than LMXBs, though it is likely that a select few LMXBs can be found at these periods. Indeed, qLMXBs at these periods should be optically brighter, having larger Roche Lobe filling companions, and should be detected as variables at a much higher efficiency than shorter period qLMXBs. $\mathrm{X}$-ray detected short period $\mathrm{BH}$ qLMXBs are likely fainter in the $\mathrm{X}$-ray than the assumed X-ray luminosity in Jonker et al. (2011) because energy is carried either through the event horizon by ADAFs (Narayan et al. 1997; Garcia et al. 2001; Hameury et al. 2003; Narayan \& McClintock 2008) or away by jets (Fender

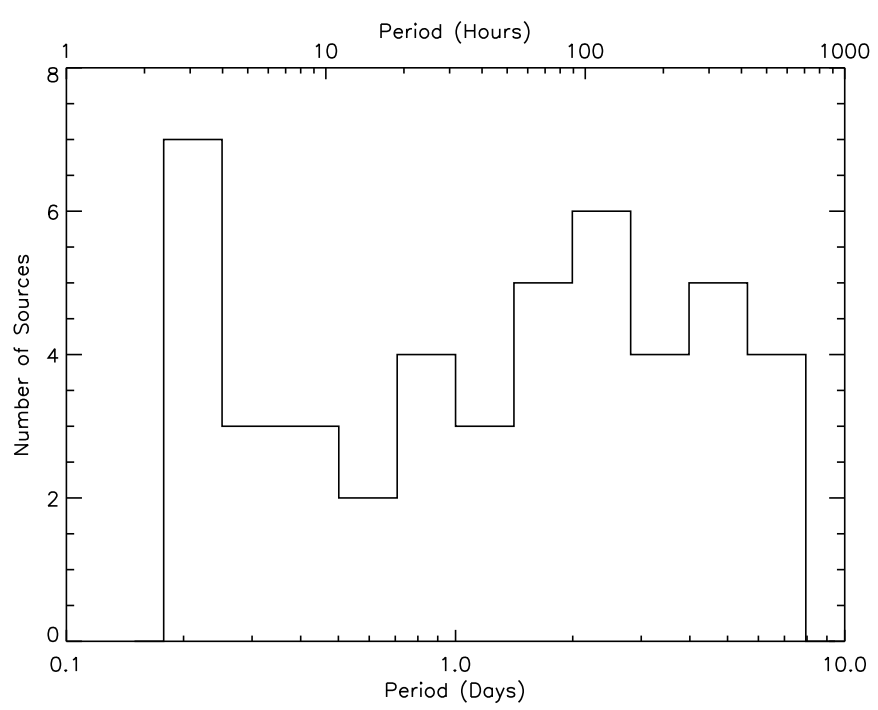

Figure 17. Period distribution of likely counterparts to X-ray sources in the GBS. There are only 70 sources for which we suspect or confirm periods, compared to the 165 sources seen to be variable. This includes 18 sources which are suspected through overlap with OGLE IV fields (Udalski et al. 2012) to be long period variables with periods above 8 days that our observations are not sensitive to. Periods between 4 and 8 days are tentative and require confirmation from observations with a longer baseline.

et al. 2003). The optical light, however, emanates from the accretion disk and donor star and is comparable to NS qLMXBs at the same periods. Therefore, X-ray detected BH qLMXBs should be nearer and therefore optically brighter and detected more efficiently as variables than may be reflected in the rough population estimate provided here.

\section{DISCUSSION}

\subsection{Likely Composition of X-Ray Sources with Nonvariable Counterparts}

Assuming 10,000 LMXBs in the Galaxy with $L_{X}=$ $10^{33} \mathrm{erg} \mathrm{cm}^{-2} \mathrm{~s}^{-1}$ and a mean $r^{\prime}$ absolute magnitude of five, Jonker et al. (2011) predicted that the GBS should find 120 qLMXBs with optical counterparts with $i^{\prime}<23$, which translates to $71 \mathrm{qLMXBs}$ with $r^{\prime}<23$, with 53 lying in the region imaged by Mosaic-II. These are mostly expected to be quite faint, with only 18 brighter than magnitude 21.5 . Using the known population as a guide, we expect these to have significant contributions in optical wavelengths from the light of their companion stars, and so to show ellipsoidal variations with periods of typically several hours. The amplitude of these variations depends upon $i$, being at maximum when $i=90^{\circ}$ and zero when $i=0^{\circ}$, while sources are distributed on the sky uniformly in $\cos i$. For a random distribution of orbital inclinations, half of this population has $i>60^{\circ}$, so most should show measurable ellipsoidal variations. For intrinsic variations of amplitude $0.2 \mathrm{mag}$, for example, $82 \%$ of sources would have $\Delta m>0.05 \mathrm{mag}$. For intrinsic variations $0.1 \mathrm{mag}$ in amplitude, $59 \%$ of sources would have $\Delta m>0.05$ mag. Our discovery efficiency is quite high for eclipsing sources and even small amplitude flickering and periodic sources down to $r^{\prime} \approx 21.5$ (Ratti et al. 2013; Britt et al. 2013; Torres et al. 2014). Some portion of counterparts with no detected optical variability will be high inclination systems, but it is not possible that inclination effects account for a majority of the $567 \mathrm{X}$-ray sources with candidate counterparts that do not vary. An example of finding charts and 

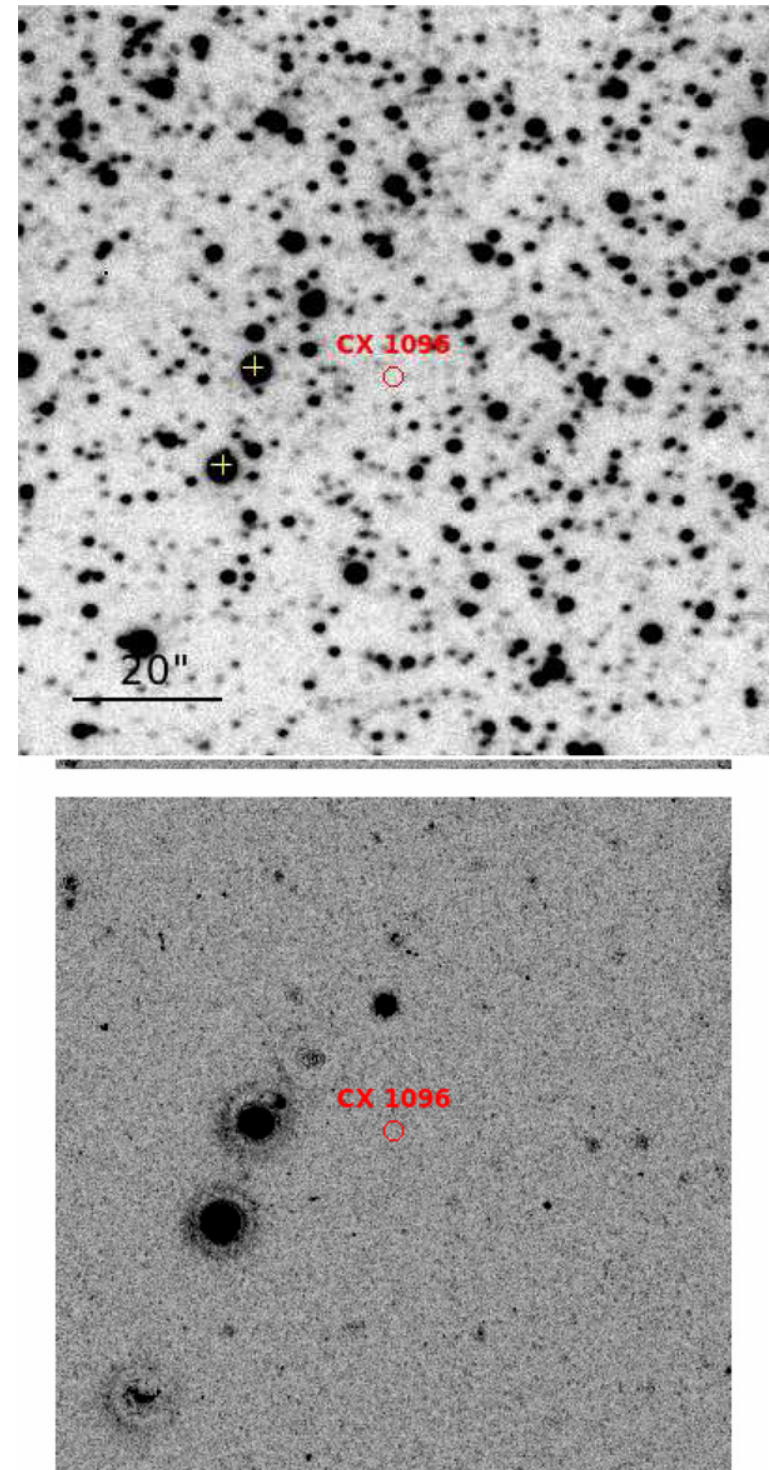

Figure 18. Top: finder chart for CX1096 with X-ray position plotted in red. Bottom: variance map of Mosaic-II images of this field. Similar figures for each source without a detected variable star coincident with the X-ray position are available in the online journal.

(The complete figure set (1138 images) and a color version are available in the online journal.)

variance maps for these regions is shown in Figure 18 and the same figure for all sources is available in the online version of the article. Still, it is difficult to place constraints on the make-up of these non-variables because we have spectroscopically confirmed that some systems that we would expect to show either flickering or ellipsoidal modulations do not, in fact, vary at all (e.g., Britt et al. 2013, CX561). Given our X-ray detection limit of 3 photons, our sensitivity to AGNs is an unabsorbed flux in the $2-10 \mathrm{keV}$ range of $\approx 5 \times 10^{-14} \mathrm{erg} \mathrm{s}^{-1} \mathrm{~cm}^{-2}$ assuming an average $N_{\mathrm{H}}$ of $2 \times 10^{22} \mathrm{~cm}^{-2}$ and a photon index of $\Gamma=1.7$. From the observed distribution of AGN luminosities in Giacconi et al. (2001), we estimate there to be $\approx 590$ AGNs in the Mosaic-II region at this flux. Our observations are slightly softer, from $0.3-8 \mathrm{keV}$, so that X-ray extinction is worse, though the number of AGNs in our X-ray sample is still likely in the hundreds. As discussed in Section 3, there are 256 X-ray sources which both contain no variable stars and have error regions large

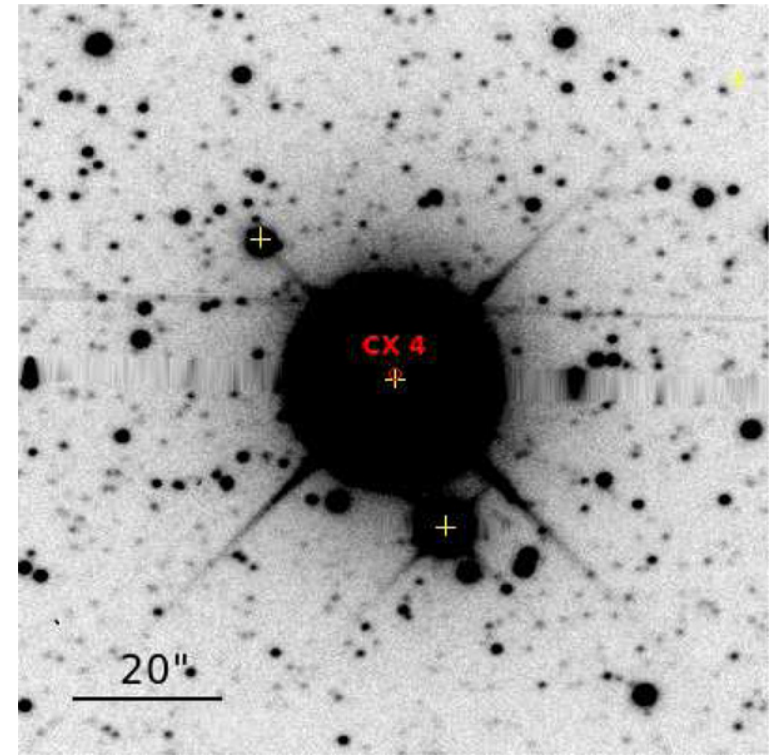

Figure 19. Finder chart for CX4 with X-ray position plotted in red. This X-ray source was classified as a G9III star by Hynes et al. (2012). Similar figures for each saturated star coincident with the X-ray position are available in the online journal.

(The complete figure set (353 images) and a color version are available in the online journal).

enough to admit likely interlopers. It is likely that many of these "non-variables" are in fact background AGNs with no optical counterpart coincident with field stars.

Saturated stars in this survey generally have very low X-ray to optical flux ratios, and many nearby active stars in the GBS will saturate in Mosaic-II data. An example of a bright star saturating in these data is shown in Figure 19.

\subsection{Likely Composition of Periodic Counterparts to X-Ray Sources}

We have not found $\sim 11$ systems with $r^{\prime}<21$ and clear ellipsoidal variations with periods below a day as predicted, or $\sim 18$ systems with $r^{\prime}<21.5$. There are only 5 systems which show clear ellipsoidal variations with or without eclipses, are unlikely to be W UMas, and that are not dynamically confirmed CVs (CX70, CX750, CX774, CX1060, CX1167), though there could be others where the donor's gravity darkened inner face is heated through reprocessing of X-rays enough to make the minima at $\phi=0$ and $\phi=0.5$ appear to be of equal depths. There is a spike in the histogram at period of 3-4 hr, as is expected for $\mathrm{CVs}$ and the suspected population of short period qLMXBs, containing 7-10 sources. The sources with periods above 1 day should be largely RS CVns, though there are a few possible qLMXBs in this region as well, e.g., CX855 (see Section 4.29). Most RS CVns in the GBS are saturated in Mosaic-II data, as extrapolated from the plot of $\mathrm{X}$-ray versus optical luminosity shown in Figure 20 in conjunction with the predictions in Jonker et al. (2011). The fainter tail of the distribution is what we pick up in our Mosaic-II data. Immediately of note in the differences between Figure 20 and the predictions in Figure 2 are the lack of X-ray bright LMXBs. There are only 2 active LMXBs in the GBS, CX1 and CX3. CX1 is one of the 20 sources that falls off of the chip in every Mosaic-II observation. CX3 is optically faint and near brighter stars, and no significant variability could be recovered for it. The one object above $F_{X}=2 \times 10^{-12} \mathrm{erg} \mathrm{cm}^{-2} \mathrm{~s}^{-1}$ that 

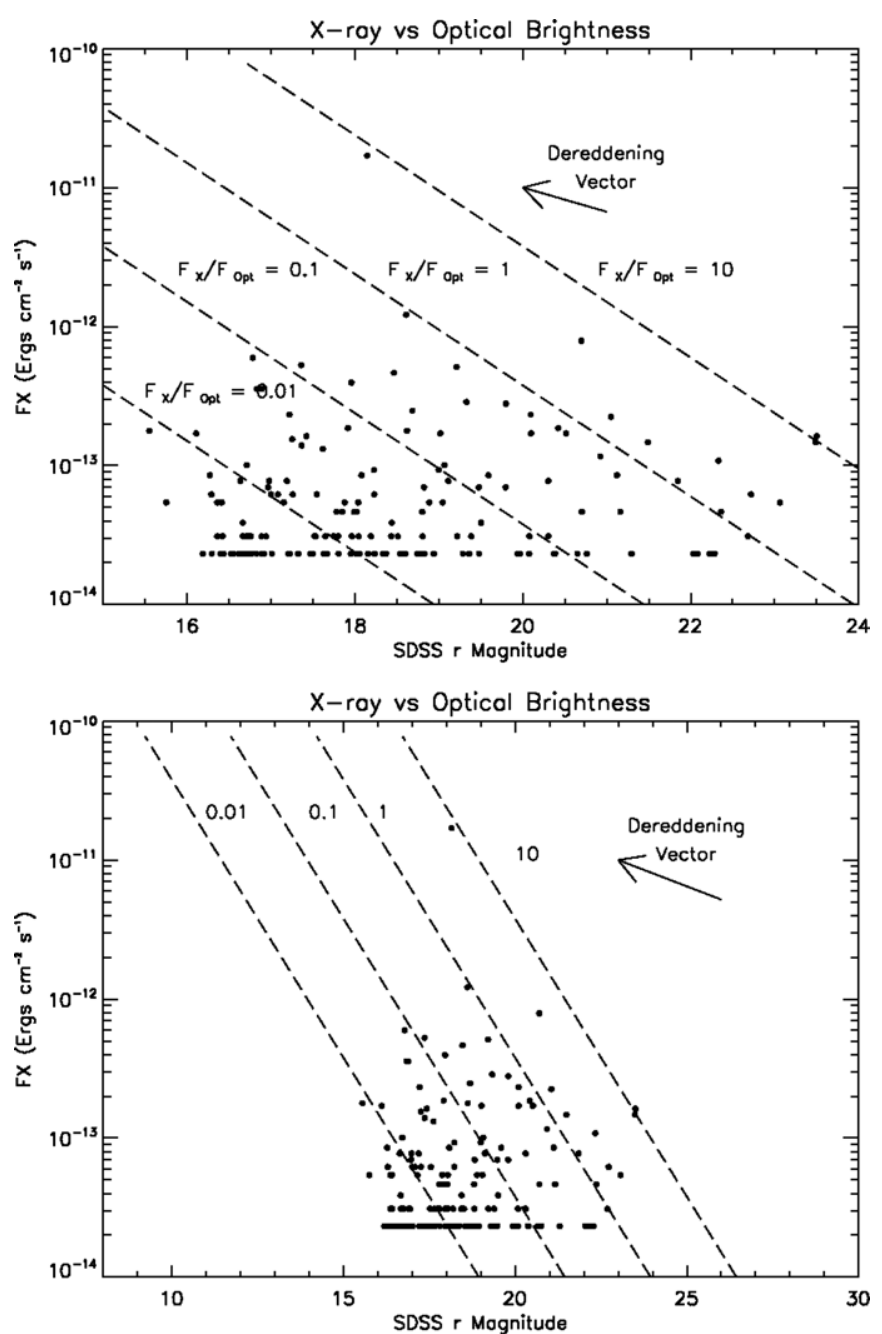

Figure 20. Each variable coincident with an X-ray source is plotted as magnitude vs. flux. The observed values are plotted, with the direction of dereddening indicated by the arrow. The length of the arrow is arbitrary, as the amount of reddening at bulge distance is heavily dependent on the line of sight. Each optical magnitude carries a systematic uncertainty of $0.5 \mathrm{mag}$ as they were calibrated with the USNO catalog. Statistical errors for the X-ray flux are given as $\sigma_{N}=1+\sqrt{N+0.75}$ (Gehrels 1986), though there are additional systematic uncertainties in the X-ray spectral shape. Uncertainties in extinction affect both $\mathrm{X}$-ray and optical flux, with reddening moving sources in the direction indicated. The same information is plotted above and below, but the scale in the bottom figure has been matched to the population synthesis in Jonker et al. (2011) in order to aid the reader in making comparisons.

appears as a variable is CX2, an AGN (Maccarone et al. 2012). We also have contamination from W UMas, which have periods $<1$ day and these sources show variations in their light curves that mimic ellipsoidal variations. Short period ellipsoidal variations with superposed flickering or night to night variations in level are likely to be accreting compact binaries. W UMas likely comprise a large number of sources with periods below a day, but in some cases they cannot be readily distinguished from compact binaries without spectroscopy, especially since BH qLMXBs can have quite low X-ray to optical flux ratios. With the large errors in estimating this ratio, it is not necessarily a reliable diagnostic on its own for differentiating between $\mathrm{BH}$ qLMXBs showing ellipsoidal variations and W UMas.

Though individual sources are difficult to classify exactly without spectroscopy, we can make probabalistic statements about the population as a whole. The total number of GBS X-ray sources closely matches the number predicted by the population model in Jonker et al. (2011). That population model also predicted that $\sim 400$ sources in the region covered by Mosaic-II would not have an optical counterpart in our variability survey. We found 111 sources without any visible star inside the X-ray error circle in Sloan $r^{\prime}$, while a further 567 objects had at least one star inside the error circle which was not seen to vary. It is certain, given the stellar density in the region, that many of these non-variable stars are not the optical counterpart to the $\mathrm{X}$-ray source, but rather are field stars. The crowding in our fields in the bulge region is, on average, high enough that coincident alignments are expected, especially for sources observed in the $\mathrm{X}$-ray at a large off-axis angle. Some X-ray sources observed far off-axis and with few counts have confidence regions $10^{\prime \prime}$ across, with tens of candidate counterparts. Two hundred and fiftysix X-ray sources have an uncertainty in the position of $\geqslant 1^{\prime \prime} .9$, which admits probable chance alignment with at least one field star. It is therefore possible that the expected remaining sources without optical counterparts, primarily AGN and including many UCXBs, IPs, and qLMXBs could be "hiding" in the group of non-variables. Still, some non-variable objects have been identified through spectroscopy to be interacting binaries, e.g., CX561 (Britt et al. 2013) and CX1004 (Torres et al. 2014). It is also possible that these objects are in fact variable, but less than what we can detect at a level considered significant here.

\subsection{Active Stars}

RS CVns are much more common than LMXBs. They are comparatively well studied, and there are far fewer uncertainties in estimates of their population compared to qLMXBs. Jonker et al. (2011) predicted $\approx 447 \mathrm{RS}$ CVns in the GBS area covered by Mosaic-II, all with optical counterparts as they are relatively optically bright and X-ray faint. They also show no detectable intrinsic aperiodic variations, apart from occasional flares, but instead a smooth light curve that is a combination of variations on the same period produced by star spots which the Lomb-Scargle statistic is excellent at finding. We find 27 sources with periods above a day, and 18 sources with what could be periodic behavior with periods above 8 days. Based on Monte Carlo simulations with noise on the scale of relative photometric errors, we estimate that we have recovered periods for $\sim 70 \%$ of unsaturated RS CVns with periods less than 8 days and suspected long period variables, which implies that $40 \mathrm{RS}$ $\mathrm{CVns}$ are unsaturated. Referring to the population estimate in Figure 2, most RS CVns should indeed be saturated in our Mosaic-II data. Because we only have 255 sources aligned with a saturated star which are likely RS CVns (see Hynes et al. 2012), and only a further 98 sources either on bleed trails or close enough to a saturated star for photometry to be impossible, we have detected at maximum somewhat fewer RS CVns in the Mosaic-II region than the 447 expected, though the exact number fewer remains uncertain from these data.

We also find 19 candidate counterparts with periods below a day. A similar estimate to that above with W UMas shows that, since there is no instrinsic flickering in W UMa systems on a scale that we can detect as there is in CVs and qLMXBs, we expect to recover nearly all of the periods for W UMas which do not saturate. Crudely assuming that the spike in the period histogram at 3-4 hr is due entirely to either CVs or qLMXBs and that $\mathrm{W}$ UMas are responsible for all other detected periods below a day, we estimate that we observe $\sim 11$ unsaturated $\mathrm{W}$ UMa systems. Using the population estimate in Figure 2, we expect most W UMas in the GBS to saturate in Mosaic-II data. 
Also among the non-variables and saturated stars are coronally active single stars (Hynes et al. 2012). These are typically faint X-ray sources, especially compared to their optical luminosity. While most MS stars with coronal emission should have $\left(L_{X} / L_{\text {opt }}\right) \ll 1$, M dwarfs peak in luminosity in infrared wavelengths such that $L_{\mathrm{opt}} \ll L_{\mathrm{Bol}}$. It is unsurprising, therefore, that this optically deep data set contains 15 objects that appear to be flare stars, showing small flares of $<1$ mag that decay over a few hours. Some of these also show small amplitude sinusoidal periods which are consistent with sunspots on flare stars, e.g., CX161 and CX1220.

\section{4. $C V s$ and $L M X B s$}

We have 76 sources that flicker without a large outburst or periodicity. These are likely a mix of IPs, CVs, and qLMXBs. The data presented here are largely ambiguous in differentiating between $\mathrm{BH}$ and NS qLMXBs, and we treat their populations taken together as the number of $\mathrm{BH}$ qLMXBs should equal $\approx 10 \%$ of NS qLMXBs. Many IPs in this optical data set will not have visible counterparts. The initial estimate of visible IPs in the survey area is sensitive to the optical magnitude we reach. Referring to Figure 2, most X-ray detected IPs are predicted to be fainter than $r^{\prime}=23$. We have difficulty securely detecting variability below $0.2 \mathrm{mag}$ fainter than $r^{\prime}=22$, so a larger proportion of IPs may be hiding among the sources with nonvariable or non-detected counterparts. Because of their high $\mathrm{X}$-ray luminosity at high accretion rate, the sources that show dramatic aperiodic flickering of a magnitude or more are more likely to be IPs than quiescent systems, but even quiescent CVs and qLMXBs can show large amounts of flickering (Jonker et al. 2008).

Eight counterparts to X-ray sources in our Mosaic-II data were observed to undergo what appear to be dwarf nova outbursts. These are CX18, CX39, CX81, CX87, CX298, CX426, CX476, and CX860. One of these, CX18, was confirmed through follow up photometric data and with spectroscopic observations (Britt et al. 2013). A ninth object, CX982, shows a candidate dwarf nova, but the unusual morphology of this object's light curve demands confirmation.

Quiescent CVs and qLMXBs can also show flickering, though $\mathrm{CVs}$ and LMXBs in quiescence can also have very smooth light curves, for example in the case of CX93 (Ratti et al. 2013). Most of these systems should have a period below a day, concentrated around a few to several hours (Gänsicke 2005), where indeed we see a spike in our period histogram. Under the assumption that qLMXBs show clear ellipsoidal modulations, we can place a limit of five sources (CX70, CX750, CX774, CX1060, CX1167) that could be qLMXBs in our optical data set, as one of the five sources showing ellipsoidal modulations, CX93, is confirmed as a CV. Some qLMXBs, though, are doubtless flickering objects without a recovered period. Indeed, a further 19 GBS objects discussed in this work and in Britt et al. (2013), Torres et al. (2014), and Wu et al. (2014) could potentially be qLMXBs. It is notoriously difficult to distinguish between quiescent CVs and qLMXBs, however, and these 24 objects will contain a mix of both. For 17 of these 24, spectroscopic data are still to be obtained or analyzed. (For many objects which could be candidate qLMXBs from photometry alone, spectroscopy suggests a CV; Torres et al. 2014.) Only five possible qLMXBs have evident ellipsoidal modulations, out of the $\approx 18$ qLMXBs which should be present going by Figure 2, and all five are potentially CVs as well. Although, if our baseline in these data were longer, it is likely that some sources which appear to flicker could be shown to have ellipsoidal variations underlying the flickering. For example, CX19 is periodic in OGLE-IV data but flickers enough that our observations did not independently recover a period. Following up these selected possible qLMXBs to confirm the expected presence of ellipsoidal modulations would provide a critical check for what large future surveys should detect. The simple assumption that qLMXBs should have clear ellipsoidal modulations does not hold for a large fraction of objects, at least those which are X-ray selected.

\subsection{Comparison to Population Synthesis Models}

The ratio of quiescent LMXBs to active LMXBs in Globular clusters has been observed to be $\approx 10$ (Heinke et al. 2003), though these studies may underestimate the number of qLMXBs by half (Heinke et al. 2005). There is an observed deficit of BH LMXBs in Globular clusters (Kalogera et al. 2004). The Galactic bulge may not produce qLMXBs with the same duty cycle as in Globular clusters, however, because those in Globular clusters are formed predominantly by different mechanisms than in the bulge, in addition to having older donor stars in general to binaries in the Milky Way. It is perhaps worth noting that we observe two active LMXBs in the GBS (CX1, CX3), which would imply $\sim 40$ qLMXBs in the Mosaic-II data if bulge LMXBs have the same quiescent to active proportions as those in Globular clusters, of which $\sim 10$ should be detectable in the optical at $r^{\prime}<23$.

The prediction actually used in Jonker et al. (2011) relied upon X-ray binary formation rates and lifetimes. The estimates of Jonker et al. (2011) used a formation rate of $10^{-5} \mathrm{NS}$ binaries formed per year (Portegies Zwart et al. 1997; Kiel \& Hurley 2006). Using a typical lifetime for LMXB systems of $1 \mathrm{Gyr}$ yields $10^{4}$ LMXB systems in the Galaxy (Jonker et al. 2011), which combined with 140 active LMXBs at a given time, yields a ratio of quiescent to active LMXBs of 70 . Pfahl et al. (2003) predict $\approx 10^{3}-10^{5}$ LMXBs by assuming many LMXBs are descendants of intermediate mass X-ray binaries, with the major driver of the different population sizes being a substantial dependence on the structure of the common envelope (CE). The population estimate in Jonker et al. (2011) fits in the center of this range. The period distribution predicted by Pfahl et al. (2003) for the median CE parameter peaks around $\log P_{\text {orb }}($ days $)=0.25$, which, referring to Figure 16, we are only likely to recover with flickering of $20 \%$ of the amplitude of orbital variability or less.

The favored population model of Kiel \& Hurley (2006), however, predicts a somewhat lower number of 1900 LMXBs, both active and quiescent, with a ratio between the two closer to 13 . The primary differences between the models in Kiel \& Hurley (2006) and Pfahl et al. (2003) are an updated model for the CE, an inclusion of the helium star's mass-loss from wind after the CE phase, metallicity of the binary, and the inclusion of tidal forces in the code. The resulting population of LMXBs would lead to $\sim 17$ qLMXBs with optical counterparts in the GBS region surveyed with the Mosaic-II instrument $(\sim 6$ with $r^{\prime}<21.5$ ) by using the same luminosity and extinction assumptions as in Jonker et al. (2011), which matches well with the number of ellipsoidal variables identified, although they remain unconfirmed as qLMXBs in this work.

If every object that could be a qLMXB based on the photometric results presented here were indeed to turn out to be a qLMXB rather than a CV, it is still difficult to support the presence of $10^{5} \mathrm{qLMXBs}$ in the Milky Way. It is possible for the qLMXB population in the Milky Way to be somewhat larger than we find it for the following reasons. 
1. The initial estimate in Jonker et al. (2011) may have overestimated the X-ray luminosity of qLMXBs.

If quiescent neutron star LMXBs that have not undergone a recent outburst are fainter than the known population of LMXBs, which have been followed into quiescence from outburst, they might not have been detected in the X-ray.

2. Some qLMXBs with optical counterparts may be too faint to notice flickering or variability. A source with ellipsoidal modulations like the CV CX93 (Ratti et al. 2013) would likely be overlooked as a variable at $r^{\prime}=22$ because the photometric errors are on par with the amplitude of the variability. We have attempted to control for this by limiting our analysis to the predicted population with counterparts brighter than $r^{\prime}=21.5$.

3. Some qLMXBs could also be intrinsically non-variable, showing no flickering or ellipsoidal modulations. A candidate BH qLMXB based on spectroscopy, CX561, shows no variability despite being at $r^{\prime}=20$. If this is the case, our complementary spectroscopic survey (e.g., Torres et al. 2014) will be the best way to find qLMXB systems.

4. Some qLMXBs may flicker too much ( $\gtrsim 50 \%$ of periodic variations) to recover periods reliably. This cannot bring us up to a Galactic population as high as $10^{5}$ qLMXBs for several reasons. First, as shown through Monte Carlo simulations in Figure 16, we can reliably find periods below 1 day even with intrinsic flickering up to $50 \%$. Second, IPs in the survey should be flickering, and the observed number of flickering sources roughly matches the number of expected IPs. While a few qLMXBs are likely hiding in this population, there are not enough flickering sources to account for both the expected population of IPs and a large unseen population of qLMXBs. This seems at best a partial solution.

5. The initial population estimate in Jonker et al. (2011) assumed that the spatial distribution of qLMXBs followed the distribution of stars in the Milky Way. Kicks imparted to the system by both mass loss (Blaauw kicks) and by asymmetry in supernovae should increase the velocity dispersion of qLMXBs and therefore extend the scale height of the distribution above and below the Galaxy. If these kicks are large enough, the column density of qLMXBs will noticeably decline. The spatial distribution of qLMXBs above and below the Galactic plane remains poorly understood because of selection effects in the systems we have discovered, but it is clear that known systems do not exactly trace stellar density but instead more than half are found more than $0.5 \mathrm{kpc}$ above or below the plane, with almost $20 \%$ of $\mathrm{BH}$ transients found between $1 \mathrm{kpc}<z<1.5 \mathrm{kpc}$ above or below the Galactic plane (Jonker \& Nelemans 2004; Repetto et al. 2012). It is unclear whether a population discovered in quiescence would have the same observed distribution.

The lower to median theoretical estimates of $10^{3}-10^{4}$ fit reasonably well the current population as we have found it, while spectroscopic campaigns currently underway will better define the discovered population of qLMXBs in the Milky Way.

This work was supported by the National Science Foundation under grant No. AST-0908789, by the Louisiana Board of Regents Fellowship, by the NAS/Louisiana Board of Regents grant NNX07AT62A/LEQSF(2007-2010) Phase 3-02. P.G.J. and M.A.P.T. acknowledge support from the Netherlands Organisation for Scientific Research. This research has made use of NASA's Astrophysics Data System Bibliographic Services and of SAOImage DS9, developed by Smithsonian Astrophysical Observatory. T.J.M. thanks Reba Bandyopadhyay for useful discussions, especially illustrating the possibility of SyXBs.

\section{REFERENCES}

Alard, C. 2000, A\&AS, 144, 363

Alard, C., \& Lupton, R. H. 1998, ApJ, 503, 325

Bohlin, R. C., Savage, B. D., \& Drake, J. F. 1978, ApJ, 224, 132

Britt, C. T., Torres, M. A. P., Hynes, R. I., et al. 2013, ApJ, 769, 120

Cardelli, J. A., Clayton, G. C., \& Mathis, J. S. 1989, ApJ, 345, 245

Collins, D. J., \& Wheatley, P. J. 2010, MNRAS, 402, 1816

Corral-Santana, J. M., Casares, J., Muñoz-Darias, T., et al. 2013, Sci, 339, 1048

DeWitt, C., Bandyopadhyay, R. M., Eikenberry, S. S., et al. 2010, ApJ, 721,1663

Ducati, J. R., Bevilacqua, C. M., Rembold, S. B., \& Ribeiro, D. 2001, ApJ, 558,309

Fender, R. P., Gallo, E., \& Jonker, P. G. 2003, MNRAS, 343, L99

Frank, J., King, A., \& Raine, D. J. 2002, Accretion Power in Astrophysics (3rd ed.; Cambridge: Cambridge Univ. Press)

Gänsicke, B. T. 2005, in ASP Conf. Ser. 330, The Astrophysics of Cataclysmic Variables and Related Objects, ed. J.-M. Hameury \& J.-P. Lasota (San Francisco, CA: ASP), 3

Garcia, M. R., McClintock, J. E., Narayan, R., et al. 2001, ApJL, 553, L47

Gehrels, N. 1986, ApJ, 303, 336

Giacconi, R., Rosati, P., Tozzi, P., et al. 2001, ApJ, 551, 624

Gonzalez, O. A., Rejkuba, M., Zoccali, M., et al. 2012, A\&A, 543, A13

Greiss, S., Steeghs, D. T. H., Jonker, P. G., et al. 2014, MNRAS, 438, 2839

Grindlay, J. E., Hong, J., Zhao, P., et al. 2005, ApJ, 635, 920

Hameury, J.-M., Barret, D., Lasota, J.-P., et al. 2003, A\&A, 399, 631

Hands, A. D. P., Warwick, R. S., Watson, M. G., \& Helfand, D. J. 2004, MNRAS, 351,31

Heinke, C. O., Grindlay, J. E., \& Edmonds, P. D. 2005, ApJ, 622, 556

Heinke, C. O., Grindlay, J. E., Lugger, P. M., et al. 2003, ApJ, 598, 501

Hong, J., van den Berg, M., Schlegel, E. M., et al. 2005, ApJ, 635, 907

Hynes, R. I., Brien, K. O., Mullally, F., \& Ashcraft, T. 2009, MNRAS, 399, 281

Hynes, R. I., Torres, M. A. P., Heinke, C. O., et al. 2014, ApJ, 780, 11

Hynes, R. I., Wright, N. J., Maccarone, T. J., et al. 2012, ApJ, 761, 162

Johnson, C. C., Hynes, R. I., Britt, C. T., et al. 2014, ApJS, in press

Jonker, P. G., Bassa, C. G., Nelemans, G., et al. 2011, ApJS, 194, 18

Jonker, P. G., Miller-Jones, J. C. A., Homan, J., et al. 2012, MNRAS, 423, 3308

Jonker, P. G., \& Nelemans, G. 2004, MNRAS, 354, 355

Jonker, P. G., Torres, M. A. P., Hynes, R. I., et al. 2014, ApJS, 210, 18

Jonker, P. G., Torres, M. A. P., \& Steeghs, D. 2008, ApJ, 680, 615

Kalogera, V., King, A. R., \& Rasio, F. A. 2004, ApJL, 601, L171

Kiel, P. D., \& Hurley, J. R. 2006, MNRAS, 369, 1152

Knevitt, G., Wynn, G. A., Vaughan, S., \& Watson, M. G. 2014, MNRAS, 437, 3087

Kong, A. K. H., McClintock, J. E., Garcia, M. R., Murray, S. S., \& Barret, D. 2002, ApJ, 570, 277

Kuulkers, E., Kouveliotou, C., Belloni, T., et al. 2013, A\&A, 552, A32

Lasota, J.-P. 2008, NewAR, 51, 752

Lewin, W. H. G., \& van der Klis, M. 2006, Compact Stellar X-ray Sources (Cambridge: Cambridge Univ. Press)

Lomb, N. R. 1976, Ap\&SS, 39, 447

Lu, T.-N., Kong, A. K. H., Bassa, C., et al. 2009, ApJ, 705, 175

Lucas, P. W., Hoare, M. G., Longmore, A., et al. 2008, MNRAS, 391, 136

Maccarone, T. J., Torres, M. A. P., Britt, C. T., et al. 2012, MNRAS, 426, 3057

Mason, K. O., Seitzer, P., Tuohy, I. R., et al. 1980, ApJL, 242, L109

Mauerhan, J. C., Muno, M. P., Morris, M. R., et al. 2009, ApJ, 703, 30

Monet, D. G., Levine, S. E., Canzian, B., et al. 2003, AJ, 125, 984

Motch, C., Warwick, R., Cropper, M. S., et al. 2010, A\&A, 523, A92

Muno, M. P., Baganoff, F. K., Bautz, M. W., et al. 2003, ApJ, 599, 465

Narayan, R., Garcia, M. R., \& McClintock, J. E. 1997, ApJL, 478, L79

Narayan, R., \& McClintock, J. E. 2008, NewAR, 51, 733

Patterson, J., \& Raymond, J. C. 1985, ApJ, 292, 535

Peacock, M. B., Maccarone, T. J., Waters, C. Z., et al. 2009, MNRAS, 392, L55

Pfahl, E., Rappaport, S., \& Podsiadlowski, P. 2003, ApJ, 597, 1036

Pooley, D., \& Hut, P. 2006, ApJL, 646, L143

Pooley, D., Lewin, W. H. G., Anderson, S. F., et al. 2003, ApJL, 591, L131

Portegies Zwart, S. F., Verbunt, F., \& Ergma, E. 1997, A\&A, 321, 207

Ratti, E. M., van Grunsven, T. F. J., Jonker, P. G., et al. 2013, MNRAS, 428,3543

Rea, N., Jonker, P. G., Nelemans, G., et al. 2011, ApJL, 729, L21

Repetto, S., Davies, M. B., \& Sigurdsson, S. 2012, MNRAS, 425, 2799 
Scargle, J. D. 1982, ApJ, 263, 835

Schandl, S., Meyer-Hofmeister, E., \& Meyer, F. 1997, A\&A, 318, 73

Schlegel, D. J., Finkbeiner, D. P., \& Davis, M. 1998, ApJ, 500, 525

Servillat, M., Grindlay, J., van den Berg, M., et al. 2012, ApJ, 748, 32

Shaw, R. A. 2009, NOAO Data Handbook (Version 1.1; Tucson, AZ: Univ. Arizona Press)

Skrutskie, M. F., Cutri, R. M., Stiening, R., et al. 2006, AJ, 131, 1163

Soszyński, I., Dziembowski, W. A., Udalski, A., et al. 2011a, AcA, 61, 1

Soszyński, I., Udalski, A., Pietrukowicz, P., et al. 2011b, AcA, 61, 285

Stellingwerf, R. F. 1978, ApJ, 224, 953

Stetson, P. B. 1987, PASP, 99, 191

Swank, J. H., Boldt, E. A., Holt, S. S., Rothschild, R. E., \& Serlemitsos, P. J. 1978, ApJL, 226, L133

Szymański, M. K., Udalski, A., Soszyński, I., et al. 2011, AcA, 61, 83

Torres, M. A. P., Jonker, P. G., Bassa, C. G., et al. 2014, MNRAS, 440, 365
Udalski, A., Kowalczyk, K., Soszyński, I., et al. 2012, AcA, 62, 133

van den Berg, M., Hong, J. S., \& Grindlay, J. E. 2009, ApJ, 700, 1702

van den Berg, M., Penner, K., Hong, J., et al. 2012, ApJ, 748, 31

Vaughan, S. 2010, MNRAS, 402, 307

Warner, B. 1976, in IAU Symp. 73, Structure and Evolution of Close Binary Systems, ed. P. Eggleton, S. Mitton, \& J. Whelan (Cambridge: Cambridge Univ. Press), 85

Warner, B. 2003, Cataclysmic Variable Stars (Cambridge: Cambridge Univ. Press)

Wijnands, R., Heinke, C. O., Pooley, D., et al. 2005, ApJ, 618, 883

Wu, J., Jonker, P. G., Torres, M. A. P., et al. 2014, MNRAS, submitted

Wu, Y. X., Yu, W., Li, T. P., Maccarone, T. J., \& Li, X. D. 2010, ApJ, 718,620

Zacharias, N., Finch, C., Girard, T., et al. 2009, yCat, 1315, 0

Zurita, C., Durant, M., Torres, M. A. P., et al. 2008, ApJ, 681, 1458 